\title{
Hierarchical Fixed Point Problems in Uniformly Smooth Banach Spaces
}

\author{
Lu-Chuan Ceng, ${ }^{1}$ Ching-Feng Wen, ${ }^{2}$ and Chin-Tzong Pang ${ }^{3}$ \\ ${ }^{1}$ Department of Mathematics, Shanghai Normal University and Scientific Computing Key Laboratory of \\ Shanghai Universities, Shanghai 200234, China \\ ${ }^{2}$ Center for Fundamental Science, Kaohsiung Medical University, Kaohsiung 807, Taiwan \\ ${ }^{3}$ Department of Information Management, Yuan Ze University, Chung-Li 32003, Taiwan
}

Correspondence should be addressed to Chin-Tzong Pang; imctpang@saturn.yzu.edu.tw

Received 4 November 2013; Accepted 18 December 2013; Published 22 January 2014

Academic Editor: Ngai-Ching Wong

Copyright (C) 2014 Lu-Chuan Ceng et al. This is an open access article distributed under the Creative Commons Attribution License, which permits unrestricted use, distribution, and reproduction in any medium, provided the original work is properly cited.

We propose some relaxed implicit and explicit viscosity approximation methods for hierarchical fixed point problems for a countable family of nonexpansive mappings in uniformly smooth Banach spaces. These relaxed viscosity approximation methods are based on the well-known viscosity approximation method and hybrid steepest-descent method. We obtain some strong convergence theorems under mild conditions.

\section{Introduction}

Let $X$ be a real Banach space and $U$ the unit sphere of $X$; that is, $U=\{x \in X:\|x\|=1\}$. Recall that $X$ is said to be smooth if the limit

$$
\lim _{t \rightarrow 0} \frac{\|x+t y\|-\|x\|}{t}
$$

exists for all $x, y \in U$; in this case, $X$ is also said to have a Gâteaux differentiable norm. $X$ is said to have a uniformly Gâteaux differentiable norm if for each $y \in U$, the limit is attained uniformly for $x \in U$. Moreover, it is said to be uniformly smooth if this limit is attained uniformly for $x, y \in$ $U$. The norm of $X$ is said to be the Fréchet differential if for each $x \in U$, this limit is attained uniformly for $y \in U$. In addition, we define a function $\rho:[0, \infty) \rightarrow[0, \infty)$ called the modulus of smoothness of $X$ as follows:

$$
\begin{gathered}
\rho(\tau)=\sup \left\{\frac{1}{2}(\|x+y\|+\|x-y\|)-1: x, y \in X,\right. \\
\|x\|=1,\|y\|=\tau\} .
\end{gathered}
$$

It is known that $X$ is uniformly smooth if and only if $\lim _{\tau \rightarrow 0} \rho(\tau) / \tau=0$.
Let $X$ be a real Banach space and let $J$ denote the normalized duality mapping from $X$ to $2^{X^{*}}$ given by

$$
J(x)=\left\{x^{*} \in X^{*}:\left\langle x, x^{*}\right\rangle=\|x\|^{2}=\left\|x^{*}\right\|^{2}\right\}, \quad \forall x \in X,
$$

where $X^{*}$ denotes the dual space of $X$ and $\langle\cdot, \cdot\rangle$ denotes the generalized duality pairing. We use $\operatorname{Fix}(T)$ to denote the set of fixed points of the mapping $T$. It is well known that if $X$ is smooth, then $J$ is single-valued and norm-to-weak ${ }^{*}$ continuous, whereas if $X$ is a Banach space with a uniformly Gâteaux differentiable norm, then $J$ is single-valued and norm-to-weak ${ }^{*}$ uniformly continuous on bounded subsets of $X$. Further, if $X$ is a uniformly smooth Banach space, then $J$ is single-valued and norm-to-norm uniformly continuous on bounded subsets of $X$. In what follows, we still denote by $J$ the single-valued normalized duality mapping.

Let $C$ be a nonempty closed convex subset of $X$. Recall that a mapping $T: C \rightarrow C$ is said to be $L$-Lipschitzian if there exists a constant $L>0$ such that

$$
\|T x-T y\| \leq L\|x-y\|, \quad \forall x, y \in C .
$$


In particular, if $L=1$, then $T$ is said to be nonexpansive; that is,

$$
\|T x-T y\| \leq\|x-y\|, \quad \forall x, y \in C
$$

We use the notation $\rightarrow$ to indicate the weak convergence and the one $\rightarrow$ to indicate the strong convergence.

Definition 1. Let $A: C \rightarrow X$ be a mapping of $C$ into $X$. Then $A$ is said to be

(i) accretive if for each $x, y \in C$, there exists $j(x-y) \in$ $J(x-y)$ such that

$$
\langle A x-A y, j(x-y)\rangle \geq 0,
$$

where $J$ is the normalized duality mapping;

(ii) $\alpha$-strongly accretive if for each $x, y \in C$, there exists $j(x-y) \in J(x-y)$ such that

$$
\langle A x-A y, j(x-y)\rangle \geq \alpha\|x-y\|^{2}
$$

for some $\alpha \in(0,1)$;

(iii) pseudocontractive if for each $x, y \in C$, there exists $j(x-y) \in J(x-y)$ such that

$$
\langle A x-A y, j(x-y)\rangle \leq\|x-y\|^{2} ;
$$

(iv) $\beta$-strongly pseudocontractive if for each $x, y \in C$, there exists $j(x-y) \in J(x-y)$ such that

$$
\langle A x-A y, j(x-y)\rangle \leq \beta\|x-y\|^{2}
$$

for some $\beta \in(0,1)$;

(v) $\lambda$-strictly pseudocontractive if for each $x, y \in C$, there exists $j(x-y) \in J(x-y)$ such that

$$
\langle A x-A y, j(x-y)\rangle \leq\|x-y\|^{2}-\lambda\|x-y-(A x-A y)\|^{2},
$$

for some $\lambda \in(0,1)$.

In a real smooth Banach space $X$ we say that an operator $A$ is strongly positive [1] if there exists a constant $\bar{\gamma}>0$ with the property

$$
\begin{gathered}
\langle A x, J(x)\rangle \geq \bar{\gamma}\|x\|^{2}, \\
\|a I-b A\|=\sup _{\|x\| \leq 1}|\langle(a I-b A) x, J(x)\rangle|, \\
\quad a \in[0,1], \quad b \in[-1,1],
\end{gathered}
$$

where $I$ is the identity mapping.

Recently, the problem of convergence of implicit iterative algorithms to a common fixed point for a family of nonexpansive mappings and its extensions to Hilbert spaces or Banach spaces have been considered by many authors; see [1-9] and the references therein.
Yao et al. [10] introduced the following Halpern-type implicit iterative algorithm,

$$
x_{n}=\alpha_{n} u+\beta_{n} x_{n-1}+\gamma_{n} T x_{n}, \quad \forall n \geq 1,
$$

and proved a strong convergence theorem under suitable conditions.

On the other hand, let $C$ be a nonempty closed convex subset of a real Hilbert space $H$ and let $A: C \rightarrow H$ be a nonlinear mapping. The classical variational inequality problem (VIP) is to find $x^{*} \in C$ such that

$$
\left\langle A x^{*}, x-x^{*}\right\rangle \geq 0, \quad \forall x \in C .
$$

If we assume that $C$ is the fixed point set of a nonexpansive mapping $T$ and $S$ is another nonexpansive mapping (not necessarily with fixed points), the problem (13) becomes the VIP of finding $x^{*} \in \operatorname{Fix}(T)$ such that

$$
\left\langle(I-S) x^{*}, x-x^{*}\right\rangle \geq 0, \quad \forall x \in \operatorname{Fix}(T),
$$

introduced first by Moudafi and Maingé in [11], which is called hierarchical fixed point problem.

In particular, whenever $\operatorname{Fix}(S) \neq \emptyset$, all elements of $\operatorname{Fix}(S)$ are solutions of VIP (14). If $S$ is a $\rho$-contraction (i.e., $\| S x-$ $S y\|\leq \rho\| x-y \|$ for some $\rho \in(0,1))$, the set of solutions of VIP (14) is a singleton and it is well known as a viscosity problem, which was first introduced by Moudafi [12] and then developed by several authors $[13,14]$.

Very recently, Cai and $\mathrm{Bu}$ [1] investigated a general hierarchical fixed point problem for a countable family of continuous pseudocontractions, which covers as a special case of the problem considered in [10]. For this purpose, they first established strong convergence of an implicit iterative scheme for solving a hierarchical fixed point problem for a continuous pseudocontractive mapping in a uniformly smooth Banach space.

In this paper, let $C$ be a nonempty closed convex subset of a uniformly smooth Banach space $X$ such that $C \pm C \subset C$. Let $T: C \rightarrow C$ be a nonexpansive mapping with $\operatorname{Fix}(T) \neq \emptyset$ and let $f: C \rightarrow C$ be a fixed contractive mapping with contractive coefficient $\beta \in(0,1)$. Let $F: C \rightarrow C$ be $\alpha$-strongly accretive and $\lambda$-strictly pseudocontractive with $\alpha+\lambda>1$ and let $A: C \rightarrow C$ be a $\bar{\gamma}$-strongly positive linear bounded operator. First of all, we introduce a relaxed implicit viscosity scheme for solving a hierarchical fixed point problem for a nonexpansive mapping $T$ :

$$
x_{t}=\left(I-\theta_{t} F\right) T x_{t}+\theta_{t}\left[f\left(x_{t}\right)-t\left(A f\left(x_{t}\right)-T x_{t}\right)\right] \text {, }
$$

where $\lim _{t \rightarrow 0} \theta_{t}=0$. It is proven that as $t \rightarrow 0,\left\{x_{t}\right\}$ converges strongly to a point $z \in \operatorname{Fix}(T)$, which is the unique solution in $\operatorname{Fix}(T)$ to the VIP:

$$
\langle(F-f) z, J(z-p)\rangle \leq 0, \quad \forall p \in \operatorname{Fix}(T) .
$$

On the other hand, let $\left\{T_{n}\right\}_{n=0}^{\infty}$ be a countable family of nonexpansive mappings from $C$ to itself such that $\Omega=$ 
$\bigcap_{i=0}^{\infty} \operatorname{Fix}\left(T_{i}\right) \neq \emptyset$. We propose a relaxed implicit viscosity iterative algorithm for solving a hierarchical fixed point problem for a countable family of nonexpansive mappings $\left\{T_{n}\right\}$ :

$$
\begin{gathered}
y_{n}=\alpha_{n} f\left(y_{n}\right)+\beta_{n} x_{n}+\left(\left(1-\beta_{n}\right) I-\alpha_{n} A\right)\left(I-\epsilon_{n} F\right) T_{n} y_{n}, \\
x_{n+1}=\sigma_{n} f\left(y_{n}\right)+\left(I-\sigma_{n} A\right) T_{n} y_{n}, \quad \forall n \geq 0,
\end{gathered}
$$

where $\left\{\alpha_{n}\right\},\left\{\beta_{n}\right\},\left\{\epsilon_{n}\right\}$, and $\left\{\sigma_{n}\right\}$ are four sequences in $(0,1)$. It is proven that under mild conditions $\left\{x_{n}\right\}$ converges strongly to a point $z \in \Omega$, which is the unique solution in $\Omega$ to the VIP:

$$
\langle(A-f) z, J(z-p)\rangle \leq 0, \quad \forall p \in \Omega .
$$

Furthermore, we also propose a relaxed explicit viscosity iterative algorithm for solving another hierarchical fixed point problem for a countable family of nonexpansive mappings $\left\{T_{n}\right\}$ :

$$
\begin{gathered}
x_{0} \in C \quad \text { chosen arbitrarily, } \\
x_{n+1}=\left(I-\beta_{n} F\right) T_{n} x_{n} \\
+\beta_{n}\left[f\left(x_{n}\right)-\alpha_{n}\left(A f\left(x_{n}\right)-T_{n} x_{n}\right)\right], \quad \forall n \geq 0,
\end{gathered}
$$

where $\left\{\alpha_{n}\right\}$ and $\left\{\beta_{n}\right\}$ are two sequences in $(0,1)$. It is proven that under appropriate assumptions, $\left\{x_{n}\right\}$ converges strongly to a point $z \in \Omega$, which is the unique solution in $\Omega$ to the VIP:

$$
\langle(F-f) z, J(z-p)\rangle \leq 0, \quad \forall p \in \Omega .
$$

The above relaxed viscosity algorithms are based on the wellknown viscosity approximation method (see, e.g., [4-6, 9]) and hybrid steepest-descent method (see, e.g., [14-17]). Our results extend, improve, supplement, and develop the recent results announced by many authors.

\section{Preliminaries}

We list some lemmas that will be used in the sequel. Lemma 2 can be found in [18]. Lemma 3 is an immediate consequence of the subdifferential inequality of the function $(1 / 2)\|\cdot\|^{2}$.

Lemma 2. Let $\left\{s_{n}\right\}$ be a sequence of nonnegative real numbers satisfying

$$
s_{n+1} \leq\left(1-\alpha_{n}\right) s_{n}+\alpha_{n} \beta_{n}+\gamma_{n}, \quad \forall n \geq 0,
$$

where $\left\{\alpha_{n}\right\},\left\{\beta_{n}\right\}$, and $\left\{\gamma_{n}\right\}$ satisfy the following conditions:

(i) $\left\{\alpha_{n}\right\} \subset[0,1], \sum_{n=0}^{\infty} \alpha_{n}=\infty$;

(ii) $\lim \sup _{n \rightarrow \infty} \beta_{n} \leq 0$;

(iii) $\gamma_{n} \geq 0(\forall n \geq 0), \sum_{n=0}^{\infty} \gamma_{n}<\infty$.

Then $\lim \sup _{n \rightarrow \infty} s_{n}=0$.

Lemma 3. In a smooth Banach space $X$, there holds the following inequality:

$$
\|x+y\|^{2} \leq\|x\|^{2}+2\langle y, J(x+y)\rangle, \quad \forall x, y \in X .
$$

Let LIM be a continuous linear functional on $l^{\infty}$ and $\left(a_{0}, a_{1}, \ldots\right) \in l^{\infty}$. We write LIM $a_{n}$ instead of $\operatorname{LIM}\left(\left(a_{0}, a_{1}, \ldots\right)\right)$. LIM is said to be Banach limit if LIM satisfies $\|\operatorname{LIM}\|=\operatorname{LIM} 1=1$ and $\operatorname{LIM} a_{n+1}=\operatorname{LIM} a_{n}$ for all $\left(a_{0}, a_{1}, \ldots\right) \in l^{\infty}$. It is well known that for Banach limit LIM the following holds:

(i) for all $n \geq 1, a_{n} \leq c_{n}$ implies that $\operatorname{LIM} a_{n} \leq \operatorname{LIM} c_{n}$;

(ii) $\operatorname{LIM} a_{n+N}=\operatorname{LIM} a_{n}$ for any fixed positive integer $N$;

(iii) $\lim \inf _{n \rightarrow \infty} a_{n} \leq \operatorname{LIM} a_{n} \leq \lim \sup _{n \rightarrow \infty} a_{n}$ for all $\left(a_{0}, a_{1}, \ldots\right) \in l^{\infty}$.

It is easy to see that there holds the following conclusion.

Lemma 4 (see [19]). Let $\left(a_{0}, a_{1}, \ldots\right) \in l^{\infty}$. If $L I M a_{n}=0$, then there exists a subsequence $\left\{a_{n_{k}}\right\}$ of $\left\{a_{n}\right\}$ such that $a_{n_{k}} \rightarrow 0$ as $k \rightarrow \infty$.

Recall that a Banach space $X$ is said to satisfy Opial's condition, if whenever $\left\{x_{n}\right\}$ is a sequence in $X$ which converges weakly to $x$ as $n \rightarrow \infty$, then

$$
\limsup _{n \rightarrow \infty}\left\|x_{n}-x\right\|<\limsup _{n \rightarrow \infty}\left\|x_{n}-y\right\|, \quad \forall y \in X, y \neq x .
$$

Lemma 5 (Demiclosedness principle; see [20, Theorem 10.3]). Let $X$ be a reflexive Banach space satisfying Opial's condition, $C$ a nonempty closed convex subset of $X$, and $T: C \rightarrow C$ a nonexpansive mapping. Then the mapping $I-T$ is demiclosed on $C$, where $I$ is the identity mapping; that is, if $\left\{x_{n}\right\}$ is a sequence of $C$ such that $x_{n} \rightarrow x$ and $(I-T) x_{n} \rightarrow y$, then $(I-T) x=y$.

The following lemma can be derived by the standard argument and hence its proof will be omitted.

Lemma 6. Let $C$ be a nonempty closed convex subset of a real smooth Banach space $X$ and let $F: C \rightarrow X$ be a mapping.

(i) If $F: C \rightarrow X$ is $\alpha$-strongly accretive and $\lambda$ strictly pseudocontractive with $\alpha+\lambda \geq 1$, then $I-$ $F$ nonexpansive and $F$ is Lipschitz continuous with constant $1+1 / \lambda$;

(ii) If $F: C \rightarrow X$ is $\alpha$-strongly accretive and $\lambda$-strictly pseudocontractive with $\alpha+\lambda>1$, then for any fixed $\tau \in(0,1), I-\tau F$ is contractive with coefficient $1-\tau(1-$ $\sqrt{(1-\alpha) / \lambda})$

\section{Relaxed Implicit Viscosity Scheme for Hierarchical Fixed Point Problem for a Nonexpansive Mapping}

In this section, we introduce our relaxed implicit viscosity scheme for solving hierarchical fixed point problem for a nonexpansive mapping and show the strong convergence theorem. First, we list several useful and helpful lemmas.

Lemma 7 (see [21]). Let $X$ be a Banach space, $C$ a nonempty closed and convex subset of $X$, and $T: C \rightarrow C$ a continuous 
and strong pseudocontraction. Then $T$ has a unique fixed point in $C$.

Lemma 8 (see [19]). Let $\left\{a_{n}\right\}$ be a sequence of nonnegative real numbers satisfying the property $a_{n+1} \leq\left(1-\gamma_{n}\right) a_{n}+\gamma_{n} \beta_{n}, \forall n \geq$ 0 , where $\left\{\gamma_{n}\right\} \subset(0,1)$ and $\left\{\beta_{n}\right\} \subset \mathbf{R}$ such that $(\mathrm{i}) \sum_{n=0}^{\infty} \gamma_{n}=\infty$ and (ii) $\lim \sup _{n \rightarrow \infty} \beta_{n} \leq 0$. Then $\left\{a_{n}\right\}$ converges to zero as $n \rightarrow \infty$.

Lemma 9 (see [22]). Let $C$ be a nonempty closed convex subset of a real Banach space $X$ and $T: C \rightarrow C$ a continuous pseudocontractive map. We denote $B=(2 I-T)^{-1}$. Then the following holds.

(i) The map $B$ is a nonexpansive self-mapping on $C$.

(ii) If $\lim _{n \rightarrow \infty}\left\|x_{n}-T x_{n}\right\|=0$, then $\lim _{n \rightarrow \infty}\left\|x_{n}-B x_{n}\right\|=$ 0 .

Lemma 10 (see [23]). Assume that $A$ is a strongly positive linear bounded operator on a smooth Banach space $X$ with coefficient $\bar{\gamma}>0$ and $0<\rho \leq\|A\|^{-1}$. Then $\|I-\rho A\| \leq 1-\rho \bar{\gamma}$.

We now state and prove our first result.

Theorem 11. Let $C$ be a nonempty closed convex subset of a uniformly smooth Banach space $X$ such that $C \pm C \subset C$. Let $T$ : $C \rightarrow C$ be a nonexpansive mapping with $\operatorname{Fix}(T) \neq \emptyset$ and $F$ : $C \rightarrow C \alpha$-strongly accretive and $\lambda$-strictly pseudocontractive with $\alpha+\lambda>1$. Let $f: C \rightarrow C$ be a fixed contractive mapping with contractive coefficient $\beta \in\left(0, \gamma_{0}\right), \gamma_{0}=1-\sqrt{(1-\alpha) / \lambda}$. Let $A: C \rightarrow C$ be a $\bar{\gamma}$-strongly positive linear bounded operator with $\bar{\gamma} \beta<1$. Let $\left\{x_{t}\right\}$ be defined by

$$
x_{t}=\left(I-\theta_{t} F\right) T x_{t}+\theta_{t}\left[f\left(x_{t}\right)-t\left(A f\left(x_{t}\right)-T x_{t}\right)\right] \text {, }
$$

where $\lim _{t \rightarrow 0} \theta_{t}=0$. Then, as $t \rightarrow 0,\left\{x_{t}\right\}$ converges strongly to some fixed point $z$ of $T$, which is the unique solution in Fix $(T)$ to the VIP:

$$
\langle(F-f) z, J(z-p)\rangle \leq 0, \quad \forall p \in \operatorname{Fix}(T) .
$$

Proof. First, we claim that $\gamma_{0}<\alpha$. Indeed, it is known that strongly accretive constant $\alpha \in(0,1)$ and strictly pseudocontractive constant $\lambda \in(0,1)$. Moreover, observe that

$$
\sqrt{(1-\alpha) \lambda}<1 \Longleftrightarrow 1-\alpha<\sqrt{\frac{1-\alpha}{\lambda}} \Longleftrightarrow \gamma_{0}<\alpha .
$$

Let us show that the net $\left\{x_{t}\right\}$ is defined well. As a matter of fact, we define the mapping $S_{t}: C \rightarrow C$ as follows:

$$
S_{t} x=\left(I-\theta_{t} F\right) T x+\theta_{t}[f(x)-t(A f(x)-T x)], \quad \forall x \in C .
$$

Since $\lim _{t \rightarrow 0} \theta_{t}=0$, we may assume, without loss of generality, that $\theta_{t} \in(0,1)$ for all $t \in\left(0, \epsilon_{0}\right)$, where $\epsilon_{0}=$ $\min \left\{\left(\gamma_{0}-\beta\right) / 2(1-\bar{\gamma} \beta),\|A\|^{-1}\right\}$. Utilizing Lemmas 6 and 10, we obtain that for each $t \in\left(0, \epsilon_{0}\right)$

$$
\begin{aligned}
& \left\langle S_{t} x-S_{t} y, J(x-y)\right\rangle \\
& =\left\langle\left(I-\theta_{t} F\right) T x-\left(I-\theta_{t} F\right) T y, J(x-y)\right\rangle \\
& +\theta_{t}\langle(I-t A) f(x)-(I-t A) f(y), J(x-y)\rangle \\
& +\theta_{t} t\langle T x-T y, J(x-y)\rangle \\
& \leq\left\|\left(I-\theta_{t} F\right) T x-\left(I-\theta_{t} F\right) T y\right\|\|x-y\| \\
& +\theta_{t}\|(I-t A) f(x)-(I-t A) f(y)\|\|x-y\| \\
& +\theta_{t} t\|T x-T y\|\|x-y\| \\
& \leq\left(1-\theta_{t}\left(1-\sqrt{\frac{1-\alpha}{\lambda}}\right)\right)\|T x-T y\|\|x-y\| \\
& +\theta_{t}(1-t \bar{\gamma})\|f(x)-f(y)\|\|x-y\|+t \theta_{t}\|x-y\|^{2} \\
& \leq\left(1-\theta_{t} \gamma_{0}\right)\|x-y\|^{2}+\theta_{t}(1-t \bar{\gamma}) \beta\|x-y\|^{2} \\
& +t \theta_{t}\|x-y\|^{2} \\
& =\left[1-\theta_{t}\left(\gamma_{0}-\beta-t(1-\bar{\gamma} \beta)\right)\right]\|x-y\|^{2} \\
& \leq\left[1-\theta_{t}\left(\gamma_{0}-\beta-\frac{\gamma_{0}-\beta}{2(1-\bar{\gamma} \beta)}(1-\bar{\gamma} \beta)\right)\right]\|x-y\|^{2} \\
& =\left(1-\frac{1}{2} \theta_{t}\left(\gamma_{0}-\beta\right)\right)\|x-y\|^{2} \text {. }
\end{aligned}
$$

It follows that for each $t \in\left(0, \epsilon_{0}\right), S_{t}: C \rightarrow C$ is a continuous and strongly pseudocontractive mapping with pseudocontractive coefficient $1-(1 / 2) \theta_{t}\left(\gamma_{0}-\beta\right)$. Hence, by Lemma 7 we know that there exists a unique fixed point in $C$, denoted by $x_{t}$, which uniquely solves the fixed point equation:

$$
x_{t}=\left(I-\theta_{t} F\right) T x_{t}+\theta_{t}\left[f\left(x_{t}\right)-t\left(A f\left(x_{t}\right)-T x_{t}\right)\right] \text {. }
$$

Let us show the uniqueness of the solution of VIP (25). Suppose both $z_{1} \in \operatorname{Fix}(T)$ and $z_{2} \in \operatorname{Fix}(T)$ are solutions to VIP (25). Then we have

$$
\begin{aligned}
& \left\langle(F-f) z_{1}, J\left(z_{1}-z_{2}\right)\right\rangle \leq 0, \\
& \left\langle(F-f) z_{2}, J\left(z_{2}-z_{1}\right)\right\rangle \leq 0 .
\end{aligned}
$$

Adding up the above two inequalities, we obtain

$$
\left\langle(F-f) z_{1}-(F-f) z_{2}, J\left(z_{1}-z_{2}\right)\right\rangle \leq 0
$$


Note that

$$
\begin{aligned}
\left\langle(F-f) z_{1}-(F-f) z_{2}, J\left(z_{1}-z_{2}\right)\right\rangle & \\
= & \left\langle F z_{1}-F z_{2}, J\left(z_{1}-z_{2}\right)\right\rangle \\
& -\left\langle f\left(z_{1}\right)-f\left(z_{2}\right), J\left(z_{1}-z_{2}\right)\right\rangle \\
\geq & \alpha\left\|z_{1}-z_{2}\right\|^{2}-\beta\left\|z_{1}-z_{2}\right\|^{2} \\
= & (\alpha-\beta)\left\|z_{1}-z_{2}\right\|^{2} \geq 0 .
\end{aligned}
$$

Taking into account $\alpha-\beta>0$, we have $z_{1}=z_{2}$, and hence the uniqueness is proved. We use $\widetilde{z}$ to denote the unique solution of VIP (25).

Next, we prove that $\left\{x_{t}: t \in\left(0, \epsilon_{0}\right)\right\}$ is bounded. Indeed, we note that $0<\theta_{t}<1, \forall t \in\left(0, \epsilon_{0}\right)$. Take a fixed $p \in$ $\operatorname{Fix}(T)$ arbitrarily. Utilizing Lemma 10 we deduce that for all $t \in\left(0, \epsilon_{0}\right)$

$$
\begin{aligned}
\| x_{t}- & p \|^{2} \\
= & \left\langle\left(I-\theta_{t} F\right) T x_{t}+\theta_{t}\left[f\left(x_{t}\right)-t\left(A f\left(x_{t}\right)-T x_{t}\right)\right]\right. \\
& \left.\quad-p, J\left(x_{t}-p\right)\right\rangle \\
= & \left\langle\left(I-\theta_{t} F\right) T x_{t}-\left(I-\theta_{t} F\right) T p, J\left(x_{t}-p\right)\right\rangle \\
& +\theta_{t}\left\langle(I-t A) f\left(x_{t}\right)-(I-t A) f(p), J\left(x_{t}-p\right)\right\rangle \\
& +\theta_{t} t\left\langle T x_{t}-p, J\left(x_{t}-p\right)\right\rangle-\theta_{t}\left\langle(F-f) p, J\left(x_{t}-p\right)\right\rangle \\
& +\theta_{t} t\left\langle(I-A f) p, J\left(x_{t}-p\right)\right\rangle \\
\leq & \left\|\left(I-\theta_{t} F\right) T x_{t}-\left(I-\theta_{t} F\right) T p\right\|\left\|x_{t}-p\right\| \\
& +\theta_{t}\left\|(I-t A) f\left(x_{t}\right)-(I-t A) f(p)\right\|\left\|x_{t}-p\right\| \\
& +\theta_{t} t\left\|T x_{t}-p\right\|\left\|x_{t}-p\right\|+\theta_{t}\|(F-f) p\|\left\|x_{t}-p\right\| \\
& +\theta_{t} t\|(I-A f) p\|\left\|x_{t}-p\right\| \\
& +\theta_{t} t\|(I-A f) p\|\left\|x_{t}-p\right\| \\
\leq & \left(1-\theta_{t} t\left\|x_{t}-p\right\|^{2}+\theta_{t}\|(F-f) p\|\left\|x_{t}-p\right\|\right. \\
& +\theta_{t} t\left\|T x_{t}-p\right\|\left\|x_{t}-p\right\|+\theta_{t}\|(F-f) p\|\left\|x_{t}-p\right\| \\
& +\theta_{t}(1-t \bar{\gamma})\left\|f\left(x_{t}\right)-f(p)\right\|\left\|x_{t}-p\right\| \\
& +\alpha-\alpha) p\|\| x_{t}-p \| \\
& +x_{t}-T p\|\| x_{t}-p \|
\end{aligned}
$$

$$
\begin{aligned}
= & {\left[1-\theta_{t}\left(\gamma_{0}-\beta-t(1-\bar{\gamma} \beta)\right)\right]\left\|x_{t}-p\right\|^{2} } \\
& +\theta_{t}\|(F-f) p\|\left\|x_{t}-p\right\|+\theta_{t} t\|(I-A f) p\|\left\|x_{t}-p\right\| \\
\leq & {\left[1-\theta_{t}\left(\gamma_{0}-\beta-\frac{\gamma_{0}-\beta}{2(1-\bar{\gamma} \beta)}(1-\bar{\gamma} \beta)\right)\right] } \\
& \times\left\|x_{t}-p\right\|^{2}+\theta_{t}\|(F-f) p\|\left\|x_{t}-p\right\| \\
& +\theta_{t} t\|(I-A f) p\|\left\|x_{t}-p\right\| \\
= & \left(1-\frac{1}{2} \theta_{t}\left(\gamma_{0}-\beta\right)\right)\left\|x_{t}-p\right\|^{2}+\theta_{t}\|(F-f) p\|\left\|x_{t}-p\right\| \\
& +\theta_{t} t\|(I-A f) p\|\left\|x_{t}-p\right\|,
\end{aligned}
$$

which immediately yields

$$
\begin{aligned}
\left\|x_{t}-p\right\| & \leq \frac{2}{\gamma_{0}-\beta}(\|(F-f) p\|+t\|(I-A f) p\|) \\
& \leq \frac{2}{\gamma_{0}-\beta}\left(\|(F-f) p\|+\|A\|^{-1}\|(I-A f) p\|\right) .
\end{aligned}
$$

Thus $\left\{x_{t}: t \in\left(0, \epsilon_{0}\right)\right\}$ is bounded.

Assume that $\left\{t_{n}\right\} \subset\left(0, \epsilon_{0}\right)$ and $t_{n} \rightarrow 0$ as $n \rightarrow \infty$. Set $\theta_{n}=\theta_{t_{n}}$ and $x_{n}:=x_{t_{n}}$, and define $\mu: C \rightarrow \mathbf{R}$ by $\mu(x)=$ $\operatorname{LIM}\left\|x_{n}-x\right\|^{2}, \forall x \in C$, where LIM is a Banach limit on $l^{\infty}$. Let

$$
K=\left\{x \in C: \mu(x)=\min _{y \in C} \operatorname{LIM}\left\|x_{n}-y\right\|^{2}\right\} .
$$

We see easily that $K$ is a nonempty closed convex subset of $X$. Note that $\left\|x_{n}-T x_{n}\right\|=\theta_{n} \| f\left(x_{n}\right)-t_{n}\left(A f\left(x_{n}\right)-T x_{n}\right)-$ $F T x_{n} \| \rightarrow 0$ as $n \rightarrow \infty$. In terms of Lemma 9, we know that the mapping $B=(2 I-T)^{-1}: C \rightarrow C$ is nonexpansive and $\operatorname{Fix}(T)=\operatorname{Fix}(B)$ and $\lim _{n \rightarrow \infty}\left\|x_{n}-B x_{n}\right\|=0$, where $I$ denotes the identity operator. It follows that

$$
\begin{aligned}
\mu(B x) & =\operatorname{LIM}\left\|x_{n}-B x\right\|^{2}=\operatorname{LIM}\left\|B x_{n}-B x\right\|^{2} \\
& \leq \operatorname{LIM}\left\|x_{n}-x\right\|^{2}=\mu(x),
\end{aligned}
$$

which implies that $B(K) \subset K$; that is, $K$ is invariant under $B$. Since a uniformly smooth Banach space has the fixed point property for nonexpansive mapping, $B$ has a fixed point, say $z \in K$. Since $z$ is also a minimizer of $\mu$ over $C$, we have that, for $t \in\left(0, \epsilon_{0}\right)$ and $x \in C$,

$$
\begin{gathered}
0 \leq \frac{\mu(z+t(x-F z))-\mu(z)}{t} \\
=\operatorname{LIM} \frac{\left\|x_{n}-z+t(F z-x)\right\|^{2}-\left\|x_{n}-z\right\|^{2}}{t} \\
=\operatorname{LIM}\left(\left(\left\langle x_{n}-z, J\left(x_{n}-z+t(F z-x)\right)\right\rangle\right.\right. \\
+t\left\langle F z-x, J\left(x_{n}-z+t(F z-x)\right)\right\rangle \\
\left.\left.-\left\|x_{n}-z\right\|^{2}\right) t^{-1}\right) .
\end{gathered}
$$


Since $X$ is uniformly smooth, we conclude that the duality mapping $J$ is norm-to-norm uniformly continuous on any bounded subset of $X$. Letting $t \rightarrow 0$, we find that the two limits above can be interchanged and obtain

$$
\operatorname{LIM}\left\langle x-F z, J\left(x_{n}-z\right)\right\rangle \leq 0, \quad \forall x \in C .
$$

On the other hand, we have

$$
\begin{aligned}
x_{n} & -z \\
= & \left(I-\theta_{n} F\right) T x_{n}-\left(I-\theta_{n} F\right) T z \\
& +\theta_{n}\left[\left(I-t_{n} A\right) f\left(x_{n}\right)-\left(I-t_{n} A\right) f(z)+t_{n}\left(T x_{n}-z\right)\right] \\
& +\theta_{n}(f-F) z+\theta_{n} t_{n}(I-A f) z .
\end{aligned}
$$

It follows that

$$
\begin{aligned}
\| x_{n}- & z \|^{2} \\
= & \left\langle\left(I-\theta_{n} F\right) T x_{n}-\left(I-\theta_{n} F\right) T z, J\left(x_{n}-z\right)\right\rangle \\
& +\theta_{n}\left[\left\langle\left(I-t_{n} A\right)\left(f\left(x_{n}\right)-f(z)\right), J\left(x_{n}-z\right)\right\rangle\right. \\
& \left.\quad+t_{n}\left\langle T x_{n}-z, J\left(x_{n}-z\right)\right\rangle\right] \\
& +\theta_{n}\left\langle(f-F) z, J\left(x_{n}-z\right)\right\rangle \\
& +\theta_{n} t_{n}\left\langle(I-A f) z, J\left(x_{n}-z\right)\right\rangle \\
\leq & \left(1-\theta_{n} \gamma_{0}\right)\left\|T x_{n}-T z\right\|\left\|x_{n}-z\right\| \\
& +\theta_{n}\left[\left(1-t_{n} \bar{\gamma}\right)\left\|f\left(x_{n}\right)-f(z)\right\|\left\|x_{n}-z\right\|\right. \\
& \left.\quad+t_{n}\left\|T x_{n}-z\right\|\left\|x_{n}-z\right\|\right] \\
& +\theta_{n}\left\langle(f-F) z, J\left(x_{n}-z\right)\right\rangle \\
& +\theta_{n} t_{n}\|(I-A f) z\|\left\|x_{n}-z\right\| \\
\leq & \left(1-\theta_{n} \gamma_{0}\right)\left\|x_{n}-z\right\|^{2} \\
& +\theta_{n}\left\langle(f-F) z, J\left(x_{n}-z\right)\right\rangle \\
& +\theta_{n}\left[\left(1-t_{n} \bar{\gamma}\right) \beta\left\|x_{n}-z\right\|^{2}+t_{n}\left\|x_{n}-z\right\|^{2}\right] \\
& +\theta_{n}\left\langle(f-F) z, J\left(x_{n}-z\right)\right\rangle \\
& +\theta_{n} t_{n}\|(I-A f) z\|\left\|x_{n}-z\right\| \\
& \left.+\theta_{n}\left(\gamma_{0}-\beta-t_{n}(1-\bar{\gamma} \beta)\right)\right]\left\|x_{n}-z\right\|^{2} \\
& \left\langle(f-F) z, J\left(x_{n}-z\right)\right\rangle \\
& \\
& \left.+\gamma_{n}-z \| \bar{\gamma} \beta\right) \\
& \\
&
\end{aligned}
$$

$$
\begin{gathered}
=\left(1-\frac{1}{2} \theta_{n}\left(\gamma_{0}-\beta\right)\right)\left\|x_{n}-z\right\|^{2} \\
+\theta_{n}\left\langle(f-F) z, J\left(x_{n}-z\right)\right\rangle \\
\quad+\theta_{n} t_{n}\|(I-A f) z\|\left\|x_{n}-z\right\| .
\end{gathered}
$$

Therefore,

$$
\begin{aligned}
\| x_{n}- & z \|^{2} \\
\leq & \frac{2}{\gamma_{0}-\beta} \\
& \times\left(\left\langle(f-F) z, J\left(x_{n}-z\right)\right\rangle+t_{n}\|(I-A f) z\|\left\|x_{n}-z\right\|\right) .
\end{aligned}
$$

Combining (38) and (41), we get

$$
\operatorname{LIM}\left\|x_{n}-z\right\|^{2} \leq \frac{2}{\gamma_{0}-\beta} \operatorname{LIM}\left\langle(f-F) z, J\left(x_{n}-z\right)\right\rangle \leq 0,
$$

which leads to $\operatorname{LIM}\left\|x_{n}-z\right\|^{2}=0$. Hence there exists a subsequence which is still denoted as $\left\{x_{n}\right\}$ such that $x_{n} \rightarrow z$ as $n \rightarrow \infty$.

Next, we prove that $z$ solves VIP (25). Since

$$
x_{t}=\left(I-\theta_{t} F\right) T x_{t}+\theta_{t}\left[f\left(x_{t}\right)-t\left(A f\left(x_{t}\right)-T x_{t}\right)\right] \text {, }
$$

we can deduce that

$$
x_{t}-T x_{t}=\theta_{t}\left(f\left(x_{t}\right)-F T x_{t}\right)+\theta_{t} t\left(T x_{t}-A f\left(x_{t}\right)\right) .
$$

Since $T$ is nonexpansive, $I-T$ is accretive. So, from the accretivity of $I-T$, it follows that, for any fixed $p \in \operatorname{Fix}(T)$,

$$
\begin{aligned}
0 \leq & \left\langle(I-T) x_{t}-(I-T) p, J\left(x_{t}-p\right)\right\rangle \\
= & \left\langle(I-T) x_{t}, J\left(x_{t}-p\right)\right\rangle \\
= & \theta_{t}\left\langle f\left(x_{t}\right)-F T x_{t}, J\left(x_{t}-p\right)\right\rangle \\
& +\theta_{t} t\left\langle T x_{t}-A f\left(x_{t}\right), J\left(x_{t}-p\right)\right\rangle \\
= & \theta_{t}\left\langle(f-F) x_{t}, J\left(x_{t}-p\right)\right\rangle \\
& +\theta_{t}\left\langle F x_{t}-F T x_{t}, J\left(x_{t}-p\right)\right\rangle \\
& +\theta_{t} t\left\langle T x_{t}-A f\left(x_{t}\right), J\left(x_{t}-p\right)\right\rangle .
\end{aligned}
$$

This implies that

$$
\begin{aligned}
& \left\langle(F-f) x_{t}, J\left(x_{t}-p\right)\right\rangle \\
& \quad \leq\left\langle F x_{t}-F T x_{t}, J\left(x_{t}-p\right)\right\rangle+t\left\langle T x_{t}-A f\left(x_{t}\right), J\left(x_{t}-p\right)\right\rangle .
\end{aligned}
$$


Now replacing $t$ with $t_{n}$, letting $n \rightarrow \infty$, and noticing the boundedness of $\left\{T x_{t_{n}}-A f\left(x_{t_{n}}\right)\right\}$ and the fact that $F x_{t_{n}}-$ $F T x_{t_{n}} \rightarrow F z-F T z=0$ for $z \in \operatorname{Fix}(T)$, we have that

$$
\langle(F-f) z, J(z-p)\rangle \leq 0, \quad \forall p \in \operatorname{Fix}(T) .
$$

That is, $z \in \operatorname{Fix}(T)$ is a solution of VIP (25). Then $z=\widetilde{z}$. In summary, we infer that each cluster point of $\left\{x_{n}\right\}$ is equal to $z$ as $t_{n} \rightarrow 0$. This completes the proof.

\section{Relaxed Viscosity Algorithms for Hierarchical Fixed Point Problems for a Countable Family of Nonexpansive Mappings}

In this section, we propose relaxed implicit and explicit viscosity algorithms for solving hierarchical fixed point problems for a countable family of nonexpansive mappings and show strong convergence theorems. For this purpose, we will use the following lemmas in the sequel.

Lemma 12 (see [24]). Let $C$ be a nonempty closed convex subset of a Banach space $X$. Let $T_{1}, T_{2}, \ldots$ be a sequence of mappings of $C$ into itself. Suppose that $\sum_{n=1}^{\infty} \sup \left\{\left\|T_{n+1} x-T_{n} x\right\|\right.$ : $x \in C\}<\infty$. Then, for each $y \in C,\left\{T_{n} y\right\}$ converges strongly to some point of $C$. Moreover, let $T$ be a mapping of $C$ into itself defined by $T y=\lim _{n \rightarrow \infty} T_{n} y$, for all $y \in C$. Then $\lim _{n \rightarrow \infty} \sup \left\{\left\|T x-T_{n} x\right\|: x \in C\right\}=0$.

Lemma 13 (see [1, Lemma 2.6]). Let $C$ be a nonempty closed convex subset of a real Banach space $X$ which has uniformly Gateaux differentiable norm. Let $T: C \rightarrow C$ be a continuous pseudocontractive mapping with $\operatorname{Fix}(T) \neq \emptyset$ and let $f: C \rightarrow$ $C$ be a fixed Lipschitzian strongly pseudocontractive mapping with pseudocontractive coefficient $\beta \in(0,1)$ and Lipschitzian constant $L>0$. Let $A: C \rightarrow C$ be a $\bar{\gamma}$-strongly positive linear bounded operator with coefficient $\bar{\gamma}>0$. Assume that $C \pm C \subset$ $C$ and that $\left\{x_{t}\right\}$ converges strongly to $z \in \operatorname{Fix}(T)$ as $t \rightarrow 0$, where $x_{t}$ is defined by $x_{t}=t f\left(x_{t}\right)+(I-t A) T x_{t}$. Suppose that $\left\{x_{n}\right\} \subset C$ is bounded and that $\lim _{n \rightarrow \infty}\left\|x_{n}-T x_{n}\right\|=0$. Then $\lim \sup _{n \rightarrow \infty}\left\langle(f-A) z, J\left(x_{n}-z\right)\right\rangle \leq 0$.

Theorem 14. Let $C$ be a nonempty closed convex subset of a uniformly smooth Banach space $X$ such that $C \pm C \subset C$. Let $\left\{T_{i}\right\}_{i=0}^{\infty}$ be a countable family of nonexpansive mappings from $C$ to itself such that $\Omega=\bigcap_{i=0}^{\infty}$ Fix $\left(T_{i}\right) \neq \emptyset$. Let $F: C \rightarrow C$ be $\alpha$ strongly accretive and $\lambda$-strictly pseudocontractive with $\alpha+\lambda>$ 1 , and let $f: C \rightarrow C$ be a fixed contractive mapping with contractive coefficient $\beta \in(0,1)$. Let $A: C \rightarrow C$ be $a \bar{\gamma}$ strongly positive linear bounded operator with $\bar{\gamma} \in(\beta, 1+\beta)$. For arbitrarily given $x_{0} \in C$, let the sequence $\left\{x_{n}\right\}$ be generated iteratively by

$$
\begin{aligned}
y_{n}= & \alpha_{n} f\left(y_{n}\right)+\beta_{n} x_{n} \\
& +\left(\left(1-\beta_{n}\right) I-\alpha_{n} A\right)\left(I-\epsilon_{n} F\right) T_{n} y_{n}, \\
x_{n+1}= & \sigma_{n} f\left(y_{n}\right)+\left(I-\sigma_{n} A\right) T_{n} y_{n}, \quad \forall n \geq 0,
\end{aligned}
$$

where $\left\{\alpha_{n}\right\},\left\{\beta_{n}\right\},\left\{\epsilon_{n}\right\}$, and $\left\{\sigma_{n}\right\}$ are four sequences in $(0,1)$ satisfying the following conditions:

(i) $\lim _{n \rightarrow \infty} \alpha_{n}=\lim _{n \rightarrow \infty} \beta_{n}=0$;

(ii) $\lim _{n \rightarrow \infty}\left(\epsilon_{n} / \alpha_{n}\right)=0$, lim $\sup _{n \rightarrow \infty}\left(\sigma_{n} / \alpha_{n}\right)<\infty$, and $\sum_{n=0}^{\infty}\left(\alpha_{n} /\left(\alpha_{n}+\beta_{n}\right)\right)=\infty$.

Assume that $\sum_{n=0}^{\infty} \sup _{x \in D}\left\|T_{n+1} x-T_{n} x\right\|<\infty$ for any bounded subset $D$ of $C$, let $T$ be a mapping of $C$ into itself defined by $T x=\lim _{n \rightarrow \infty} T_{n} x$ for all $x \in C$, and suppose that $\operatorname{Fix}(T)=$ $\bigcap_{i=0}^{\infty} \operatorname{Fix}\left(T_{i}\right)$. Then, $\left\{x_{n}\right\}$ converges strongly to a point $z$ of $\Omega$ such that $z$ is a unique solution in $\Omega$ to the VIP:

$$
\langle(f-A) z, J(p-z)\rangle \leq 0, \quad \forall p \in \Omega
$$

Proof. By condition (i), we may assume, without loss of generality, that $\alpha_{n} \leq\left(1-\beta_{n}\right)\|A\|^{-1}$. Since $A$ is a $\bar{\gamma}$-strongly positive linear bounded operator on $C$, from (11) we have

$$
\|A\|=\sup \{|\langle A u, J(u)\rangle|: u \in C,\|u\|=1\} .
$$

Observe that

$$
\begin{aligned}
\left\langle\left(\left(1-\beta_{n}\right) I-\alpha_{n} A\right) u, J(u)\right\rangle & =1-\beta_{n}-\alpha_{n}\langle A u, J(u)\rangle \\
& \geq 1-\beta_{n}-\alpha_{n}\|A\| \\
& \geq 0 .
\end{aligned}
$$

It follows that

$$
\begin{aligned}
& \left\|\left(1-\beta_{n}\right) I-\alpha_{n} A\right\| \\
& =\sup \left\{\left\langle\left(\left(1-\beta_{n}\right) I-\alpha_{n} A\right) u, J(u)\right\rangle: u \in C,\|u\|=1\right\} \\
& =\sup \left\{1-\beta_{n}-\alpha_{n}\langle A u, J(u)\rangle: u \in C,\|u\|=1\right\} \\
& \quad \leq 1-\beta_{n}-\alpha_{n} \bar{\gamma} .
\end{aligned}
$$

Next, we show that $\left\{y_{n}\right\}$ is well defined. For each $n \geq 0$, define a mapping $S_{n}: C \rightarrow C$ by

$$
\begin{array}{r}
S_{n} x=\alpha_{n} f(x)+\beta_{n} x_{n}+\left(\left(1-\beta_{n}\right) I-\alpha_{n} A\right)\left(I-\epsilon_{n} F\right) T_{n} x, \\
\forall x \in C .
\end{array}
$$

For every $x, y \in C$, we have

$$
\begin{aligned}
& \left\langle S_{n} x-S_{n} y, J(x-y)\right\rangle \\
& =\alpha_{n}\langle f(x)-f(y), J(x-y)\rangle \\
& +\left\langle\left(\left(1-\beta_{n}\right) I-\alpha_{n} A\right)\right. \\
& \left.\times\left(\left(I-\epsilon_{n} F\right) T_{n} x-\left(I-\epsilon_{n} F\right) T_{n} y\right), J(x-y)\right\rangle
\end{aligned}
$$




$$
\begin{aligned}
\leq & \alpha_{n} \beta\|x-y\|^{2}+\left(1-\beta_{n}-\alpha_{n} \bar{\gamma}\right) \\
& \times\left\|\left(I-\epsilon_{n} F\right) T_{n} x-\left(I-\epsilon_{n} F\right) T_{n} y\right\|\|x-y\| \\
\leq & \alpha_{n} \beta\|x-y\|^{2} \\
& +\left(1-\beta_{n}-\alpha_{n} \bar{\gamma}\right)\left(1-\epsilon_{n} \gamma_{0}\right)\left\|T_{n} x-T_{n} y\right\|\|x-y\| \\
\leq & \alpha_{n} \beta\|x-y\|^{2}+\left(1-\beta_{n}-\alpha_{n} \bar{\gamma}\right)\|x-y\|^{2} \\
= & {\left[1-\beta_{n}-\alpha_{n}(\bar{\gamma}-\beta)\right]\|x-y\|^{2}, }
\end{aligned}
$$

where $\gamma_{0}=1-\sqrt{(1-\alpha) / \lambda}$. Therefore, $S_{n}$ is a continuous strong pseudocontraction for each $n \geq 0$. By Lemma 7, we see that there exists a unique fixed point $y_{n}$ for each $n \geq 0$ such that

$$
y_{n}=\alpha_{n} f\left(y_{n}\right)+\beta_{n} x_{n}+\left(\left(I-\beta_{n}\right) I-\alpha_{n} A\right)\left(I-\epsilon_{n} F\right) T_{n} y_{n} .
$$

That is, the sequence $\left\{y_{n}\right\}$ is well defined. Next, we prove that $\left\{x_{n}\right\}$ is bounded. Take a fixed $p \in \Omega$ arbitrarily. Taking into account $\lim _{n \rightarrow \infty}\left(\epsilon_{n} / \alpha_{n}\right)=0$, we may assume that there exists a constant $\tau \in(0,1)$ such that $\epsilon_{n} \leq \tau \alpha_{n}$ for all $n \geq 0$. Then we have

$$
\begin{aligned}
& \left\|y_{n}-p\right\|^{2} \\
& =\alpha_{n}\left\langle f\left(y_{n}\right)-A p, J\left(y_{n}-p\right)\right\rangle \\
& \quad+\beta_{n}\left\langle x_{n}-p, J\left(y_{n}-p\right)\right\rangle \\
& \quad+\left\langle\left(\left(1-\beta_{n}\right) I-\alpha_{n} A\right)\left(\left(I-\epsilon_{n} F\right) T_{n} y_{n}-\left(I-\epsilon_{n} F\right) p\right)\right. \\
& \left.\quad J\left(y_{n}-p\right)\right\rangle \\
& \quad-\epsilon_{n}\left\langle\left(\left(1-\beta_{n}\right) I-\alpha_{n} A\right) F p, J\left(y_{n}-p\right)\right\rangle \\
& \leq \alpha_{n}\left\langle f\left(y_{n}\right)-f(p), J\left(y_{n}-p\right)\right\rangle \\
& +\alpha_{n}\left\langle f(p)-A p, J\left(y_{n}-p\right)\right\rangle \\
& +\beta_{n}\left\|x_{n}-p\right\|\left\|y_{n}-p\right\| \\
& +\left(1-\beta_{n}-\alpha_{n} \bar{\gamma}\right)\left\|\left(I-\epsilon_{n} F\right) T_{n} y_{n}-\left(I-\epsilon_{n} F\right) p\right\| \\
& +\left(1-\beta_{n}-\alpha_{n} \bar{\gamma}\right)\left(1-\epsilon_{n} \gamma_{0}\right)\left\|T_{n} y_{n}-p\right\|\left\|y_{n}-p\right\| \\
& + \\
& +
\end{aligned}
$$

$$
\begin{aligned}
\leq & \alpha_{n} \beta\left\|y_{n}-p\right\|^{2}+\left(1-\beta_{n}-\alpha_{n} \bar{\gamma}\right)\left\|y_{n}-p\right\|^{2} \\
& +\alpha_{n}\left\langle f(p)-A p, J\left(y_{n}-p\right)\right\rangle \\
& +\beta_{n}\left\|x_{n}-p\right\|\left\|y_{n}-p\right\|+\epsilon_{n}\|F p\|\left\|y_{n}-p\right\| \\
= & \left(1-\beta_{n}-\alpha_{n}(\bar{\gamma}-\beta)\right)\left\|y_{n}-p\right\|^{2} \\
& +\beta_{n}\left\|x_{n}-p\right\|\left\|y_{n}-p\right\| \\
& +\alpha_{n}\|f(p)-A p\|\left\|y_{n}-p\right\|+\epsilon_{n}\|F p\|\left\|y_{n}-p\right\| \\
\leq & \left(1-\beta_{n}-\alpha_{n}(\bar{\gamma}-\beta)\right)\left\|y_{n}-p\right\|^{2} \\
& +\beta_{n}\left\|x_{n}-p\right\|\left\|y_{n}-p\right\| \\
& +\left(\alpha_{n}+\epsilon_{n}\right)(\|f(p)-A p\|+\|F p\|)\left\|y_{n}-p\right\| \\
\leq & \left(1-\beta_{n}-\alpha_{n}(\bar{\gamma}-\beta)\right)\left\|y_{n}-p\right\|^{2} \\
& +\beta_{n}\left\|x_{n}-p\right\|\left\|y_{n}-p\right\| \\
& +\alpha_{n}(1+\tau)(\|f(p)-A p\|+\|F p\|)\left\|y_{n}-p\right\|,
\end{aligned}
$$

which implies that

$$
\begin{aligned}
\left\|y_{n}-p\right\| \leq & \frac{\beta_{n}}{\beta_{n}+\alpha_{n}(\bar{\gamma}-\beta)}\left\|x_{n}-p\right\| \\
& +\frac{\alpha_{n}(\bar{\gamma}-\beta)}{\beta_{n}+\alpha_{n}(\bar{\gamma}-\beta)} \\
& \cdot \frac{(1+\tau)(\|f(p)-A p\|+\|F p\|)}{\bar{\gamma}-\beta} .
\end{aligned}
$$

Therefore, we have

$$
\begin{aligned}
\| & x_{n+1}-p \| \\
= & \left\|\sigma_{n} f\left(y_{n}\right)+\left(I-\sigma_{n} A\right) T_{n} y_{n}-p\right\| \\
= & \| \sigma_{n}\left(f\left(y_{n}\right)-f(p)\right)+\left(I-\sigma_{n} A\right) T_{n} y_{n} \\
& \quad-\left(I-\sigma_{n} A\right) T_{n} p+\sigma_{n}(f(p)-A p) \| \\
\leq & \sigma_{n}\left\|f\left(y_{n}\right)-f(p)\right\| \\
& +\left\|\left(I-\sigma_{n} A\right)\left(T_{n} y_{n}-T_{n} p\right)\right\|+\sigma_{n}\|f(p)-A p\| \\
\leq & \sigma_{n} \beta\left\|y_{n}-p\right\|+\left(1-\sigma_{n} \bar{\gamma}\right)\left\|y_{n}-p\right\|+\sigma_{n}\|f(p)-A p\| \\
= & \left(1-\sigma_{n}(\bar{\gamma}-\beta)\right)\left\|y_{n}-p\right\|+\sigma_{n}\|f(p)-A p\| \\
\leq & \left(1-\sigma_{n}(\bar{\gamma}-\beta)\right) \\
& \times\left[\frac{\beta_{n}}{\beta_{n}+\alpha_{n}(\bar{\gamma}-\beta)}\left\|x_{n}-p\right\|+\frac{\alpha_{n}(\bar{\gamma}-\beta)}{\beta_{n}+\alpha_{n}(\bar{\gamma}-\beta)}\right. \\
& \left.\quad \cdot \frac{(1+\tau)(\|f(p)-A p\|+\|F p\|)}{\bar{\gamma}-\beta}\right]
\end{aligned}
$$




$$
\begin{aligned}
& +\sigma_{n}\|f(p)-A p\| \\
\leq & \left(1-\sigma_{n}(\bar{\gamma}-\beta)\right) \\
& \times \max \left\{\left\|x_{n}-p\right\|, \frac{(1+\tau)(\|f(p)-A p\|+\|F p\|)}{\bar{\gamma}-\beta}\right\} \\
& +\sigma_{n}\|f(p)-A p\| \\
= & \left(1-\sigma_{n}(\bar{\gamma}-\beta)\right) \\
& \times \max \left\{\left\|x_{n}-p\right\|, \frac{(1+\tau)(\|f(p)-A p\|+\|F p\|)}{\bar{\gamma}-\beta}\right\} \\
& +\sigma_{n}(\bar{\gamma}-\beta) \frac{\|f(p)-A p\|}{\bar{\gamma}-\beta} \\
\leq & \max \left\{\left\|x_{n}-p\right\|, \frac{(1+\tau)(\|f(p)-A p\|+\|F p\|)}{\bar{\gamma}-\beta},\right. \\
& \left.\quad \frac{\|f(p)-A p\|}{\bar{\gamma}-\beta}\right\} \\
\leq & \max \left\{\left\|x_{n}-p\right\|, \frac{(1+\tau)(\|f(p)-A p\|+\|F p\|)}{\bar{\gamma}-\beta}\right\} .
\end{aligned}
$$

By induction, we get

$$
\begin{aligned}
& \left\|x_{n}-p\right\| \\
& \quad \leq \max \left\{\left\|x_{0}-p\right\|, \frac{(1+\tau)(\|f(p)-A p\|+\|F p\|)}{\bar{\gamma}-\beta}\right\},
\end{aligned}
$$$$
\forall n \geq 0 \text {. }
$$

Therefore, $\left\{x_{n}\right\}$ is bounded and so are the sequences $\left\{y_{n}\right\},\left\{T_{n} y_{n}\right\}$. We observe that

$$
\begin{aligned}
\left\|y_{n}-T_{n} y_{n}\right\|= & \| \alpha_{n}\left(f\left(y_{n}\right)-A T_{n} y_{n}\right)+\beta_{n}\left(x_{n}-T_{n} y_{n}\right) \\
& \quad \epsilon_{n}\left(\left(1-\beta_{n}\right) I-\alpha_{n} A\right) F T_{n} y_{n} \| \\
\leq & \alpha_{n}\left\|f\left(y_{n}\right)-A T_{n} y_{n}\right\|+\beta_{n}\left\|x_{n}-T_{n} y_{n}\right\| \\
& +\epsilon_{n}\left\|\left(\left(1-\beta_{n}\right) I-\alpha_{n} A\right) F T_{n} y_{n}\right\| \\
\leq & \alpha_{n}\left\|f\left(y_{n}\right)-A T_{n} y_{n}\right\|+\beta_{n}\left\|x_{n}-T_{n} y_{n}\right\| \\
& +\epsilon_{n}\left(1-\beta_{n}-\alpha_{n} \bar{\gamma}\right)\left\|F T_{n} y_{n}\right\| \\
\leq & \alpha_{n}\left\|f\left(y_{n}\right)-A T_{n} y_{n}\right\|+\beta_{n}\left\|x_{n}-T_{n} y_{n}\right\| \\
& +\epsilon_{n}\left\|F T_{n} y_{n}\right\|,
\end{aligned}
$$

which go together with condition (i) and $\epsilon_{n} \leq \tau \alpha_{n}, \forall n \geq 0$, implying that

$$
\lim _{n \rightarrow \infty}\left\|y_{n}-T_{n} y_{n}\right\|=0 .
$$

On the other hand, we have

$$
\left\|y_{n}-T y_{n}\right\| \leq\left\|y_{n}-T_{n} y_{n}\right\|+\left\|T_{n} y_{n}-T y_{n}\right\|
$$

Utilizing Lemma 12, we immediately derive

$$
\lim _{n \rightarrow \infty}\left\|y_{n}-T y_{n}\right\|=0
$$

Let $x_{t}=t f\left(x_{t}\right)+(I-t A) T x_{t}$. Utilizing [1, Lemma 2.5] and Lemma 13, we conclude that $\left\{x_{t}\right\}$ converges strongly to $z \in$ $\operatorname{Fix}(T)=\bigcap_{i=0}^{\infty} \operatorname{Fix}\left(T_{i}\right)=\Omega$ and

$$
\limsup _{n \rightarrow \infty}\left\langle(f-A) z, J\left(y_{n}-z\right)\right\rangle \leq 0 .
$$

Finally, we show that $x_{n} \rightarrow z$ as $n \rightarrow \infty$. We observe that

$$
\begin{aligned}
\| y_{n} & -z \|^{2} \\
= & \alpha_{n}\left\langle f\left(y_{n}\right)-A z, J\left(y_{n}-z\right)\right\rangle+\beta_{n}\left\langle x_{n}-z, J\left(y_{n}-z\right)\right\rangle \\
& +\left\langle\left(\left(1-\beta_{n}\right) I-\alpha_{n} A\right)\left(T_{n} y_{n}-z\right), J\left(y_{n}-z\right)\right\rangle \\
& -\epsilon_{n}\left\langle\left(\left(1-\beta_{n}\right) I-\alpha_{n} A\right) F T_{n} y_{n}, J\left(y_{n}-z\right)\right\rangle \\
\leq & \left(1-\beta_{n}-\alpha_{n} \bar{\gamma}\right)\left\|y_{n}-z\right\|^{2}+\beta_{n}\left\|x_{n}-z\right\|\left\|y_{n}-z\right\| \\
& +\alpha_{n}\left\langle f\left(y_{n}\right)-f(z), J\left(y_{n}-z\right)\right\rangle \\
& +\alpha_{n}\left\langle f(z)-A z, J\left(y_{n}-z\right)\right\rangle \\
& +\epsilon_{n}\left\|\left(\left(1-\beta_{n}\right) I-\alpha_{n} A\right) F T_{n} y_{n}\right\|\left\|y_{n}-z\right\| \\
\leq & \left(1-\beta_{n}-\alpha_{n} \bar{\gamma}\right)\left\|y_{n}-z\right\|^{2} \\
& +\beta_{n}\left\|x_{n}-z\right\|\left\|y_{n}-z\right\|+\alpha_{n} \beta\left\|y_{n}-z\right\|^{2} \\
& +\alpha_{n}\left\langle f(z)-A z, J\left(y_{n}-z\right)\right\rangle \\
& +\epsilon_{n}\left(1-\beta_{n}-\alpha_{n} \bar{\gamma}\right)\left\|F T_{n} y_{n}\right\|\left\|y_{n}-z\right\|
\end{aligned}
$$




$$
\begin{aligned}
\leq & \left(1-\beta_{n}-\alpha_{n} \bar{\gamma}\right)\left\|y_{n}-z\right\|^{2}+\frac{\beta_{n}}{2}\left\|x_{n}-z\right\|^{2}+\frac{\beta_{n}}{2}\left\|y_{n}-z\right\|^{2} \\
& +\alpha_{n} \beta\left\|y_{n}-z\right\|^{2}+\alpha_{n}\left\langle f(z)-A z, J\left(y_{n}-z\right)\right\rangle \\
& +\epsilon_{n}\left\|F T_{n} y_{n}\right\|\left\|y_{n}-z\right\| \\
= & \left(1-\frac{\beta_{n}}{2}-\alpha_{n}(\bar{\gamma}-\beta)\right)\left\|y_{n}-z\right\|^{2}+\frac{\beta_{n}}{2}\left\|x_{n}-z\right\|^{2} \\
& +\alpha_{n}\left\langle f(z)-A z, J\left(y_{n}-z\right)\right\rangle+\epsilon_{n}\left\|F T_{n} y_{n}\right\|\left\|y_{n}-z\right\|,
\end{aligned}
$$

which implies that

$$
\begin{aligned}
\left\|y_{n}-z\right\|^{2} & \\
\leq & \frac{\beta_{n}}{\beta_{n}+2 \alpha_{n}(\bar{\gamma}-\beta)}\left\|x_{n}-z\right\|^{2} \\
& +\frac{2 \alpha_{n}}{\beta_{n}+2 \alpha_{n}(\bar{\gamma}-\beta)}\left\langle f(z)-A z, J\left(y_{n}-z\right)\right\rangle \\
& +\frac{2 \epsilon_{n}}{\beta_{n}+2 \alpha_{n}(\bar{\gamma}-\beta)}\left\|F T_{n} y_{n}\right\|\left\|y_{n}-z\right\| \\
= & \left(1-\frac{2 \alpha_{n}(\bar{\gamma}-\beta)}{\beta_{n}+2 \alpha_{n}(\bar{\gamma}-\beta)}\right)\left\|x_{n}-z\right\|^{2}+\frac{2 \alpha_{n}(\bar{\gamma}-\beta)}{\beta_{n}+2 \alpha_{n}(\bar{\gamma}-\beta)} \\
& \times\left(\frac{\left\langle f(z)-A z, J\left(y_{n}-z\right)\right\rangle}{\bar{\gamma}-\beta}+\frac{\epsilon_{n}}{\alpha_{n}}\right. \\
& \left.\frac{\left\|F T_{n} y_{n}\right\|\left\|y_{n}-z\right\|}{\bar{\gamma}-\beta}\right) .
\end{aligned}
$$

Furthermore, utilizing Lemma 3 from the last relation we have

$$
\begin{aligned}
\left\|x_{n+1}-z\right\|^{2} & \\
=\| & \sigma_{n}\left(f\left(y_{n}\right)-f(z)\right)+\left(I-\sigma_{n} A\right) T_{n} y_{n} \\
& \quad-\left(I-\sigma_{n} A\right) T_{n} z+\sigma_{n}(f(z)-F(z)) \|^{2} \\
\leq & \| \sigma_{n}\left(f\left(y_{n}\right)-f(z)\right) \\
& +\left(I-\sigma_{n} A\right) T_{n} y_{n}-\left(I-\sigma_{n} A\right) T_{n} z \|^{2} \\
& +2 \sigma_{n}\left\langle f(z)-A z, J\left(x_{n+1}-z\right)\right\rangle \\
\leq & {\left[\sigma_{n} \beta\left\|y_{n}-z\right\|+\left(1-\sigma_{n} \bar{\gamma}\right)\left\|T_{n} y_{n}-T_{n} z\right\|\right]^{2} } \\
+ & 2 \sigma_{n}\|f(z)-A z\|\left\|x_{n+1}-z\right\|
\end{aligned}
$$

$$
\begin{aligned}
\leq & {\left[\sigma_{n} \beta\left\|y_{n}-z\right\|+\left(1-\sigma_{n} \bar{\gamma}\right)\left\|y_{n}-z\right\|\right]^{2} } \\
& +2 \sigma_{n}\|f(z)-A z\|\left\|x_{n+1}-z\right\| \\
= & \left(1-\sigma_{n}(\bar{\gamma}-\beta)\right)^{2}\left\|y_{n}-z\right\|^{2} \\
& +2 \sigma_{n}\|f(z)-A z\|\left\|x_{n+1}-z\right\| \\
\leq & \left\|y_{n}-z\right\|^{2}+2 \sigma_{n}\|f(z)-A z\|\left\|x_{n+1}-z\right\| \\
\leq & \left(1-\frac{2 \alpha_{n}(\bar{\gamma}-\beta)}{\beta_{n}+2 \alpha_{n}(\bar{\gamma}-\beta)}\right)\left\|x_{n}-z\right\|^{2}
\end{aligned}
$$

$$
\begin{aligned}
& +\frac{2 \alpha_{n}(\bar{\gamma}-\beta)}{\left(\beta_{n}+2 \alpha_{n}(\bar{\gamma}-\beta)\right)} \\
& \times\left(\frac{\left\langle f(z)-A z, J\left(y_{n}-z\right)\right\rangle}{\bar{\gamma}-\beta}\right. \\
& \left.+\frac{\epsilon_{n}}{\alpha_{n}} \cdot \frac{\left\|F T_{n} y_{n}\right\|\left\|y_{n}-z\right\|}{\bar{\gamma}-\beta}\right)
\end{aligned}
$$$$
+2 \sigma_{n}\|f(z)-A z\|\left\|x_{n+1}-z\right\|
$$$$
=\left(1-\frac{2 \alpha_{n}(\bar{\gamma}-\beta)}{\beta_{n}+2 \alpha_{n}(\bar{\gamma}-\beta)}\right)\left\|x_{n}-z\right\|^{2}+\frac{2 \alpha_{n}(\bar{\gamma}-\beta)}{\beta_{n}+2 \alpha_{n}(\bar{\gamma}-\beta)}
$$$$
\times\left\{\frac{\left\langle f(z)-A z, J\left(y_{n}-z\right)\right\rangle}{\bar{\gamma}-\beta}+\frac{\epsilon_{n}}{\alpha_{n}} \cdot \frac{\left\|F T_{n} y_{n}\right\|\left\|y_{n}-z\right\|}{\bar{\gamma}-\beta}\right.
$$$$
+\frac{\beta_{n}+2 \alpha_{n}(\bar{\gamma}-\beta)}{\bar{\gamma}-\beta} \cdot \frac{\sigma_{n}}{\alpha_{n}}
$$$$
\left.\cdot\|f(z)-A z\|\left\|x_{n+1}-z\right\|\right\}
$$

We note that

$$
\frac{2 \alpha_{n}(\bar{\gamma}-\beta)}{\beta_{n}+2 \alpha_{n}(\bar{\gamma}-\beta)}>\frac{2 \alpha_{n}(\bar{\gamma}-\beta)}{2 \beta_{n}+2 \alpha_{n}}=(\bar{\gamma}-\beta) \frac{\alpha_{n}}{\alpha_{n}+\beta_{n}} .
$$

Therefore, condition (ii) leads to $\sum_{n=0}^{\infty}\left(2 \alpha_{n}(\bar{\gamma}-\beta) /\left(\beta_{n}+2 \alpha_{n}(\bar{\gamma}-\right.\right.$ $\beta)))=\infty$. In addition, since $\alpha_{n} \rightarrow 0, \beta_{n} \rightarrow 0,\left(\epsilon_{n} / \alpha_{n}\right) \rightarrow 0$, and $\lim \sup _{n \rightarrow \infty}\left(\sigma_{n} / \alpha_{n}\right)<\infty$, we get the following from (64)

$$
\begin{aligned}
\limsup _{n \rightarrow \infty}\left\{\frac{\left\langle f(z)-A z, J\left(y_{n}-z\right)\right\rangle}{\bar{\gamma}-\beta}+\frac{\epsilon_{n}}{\alpha_{n}} \cdot \frac{\left\|F T_{n} y_{n}\right\|\left\|y_{n}-z\right\|}{\bar{\gamma}-\beta}\right. \\
+\frac{\beta_{n}+2 \alpha_{n}(\bar{\gamma}-\beta)}{\bar{\gamma}-\beta} \cdot \frac{\sigma_{n}}{\alpha_{n}} \\
\left.\cdot\|f(z)-F(z)\|\left\|x_{n+1}-z\right\|\right\} \leq 0 .
\end{aligned}
$$

Applying Lemma 2, we have $x_{n} \rightarrow z$ as $n \rightarrow \infty$. This completes the proof. 
Remark 15. Put $\alpha_{n}=\sigma_{n}=1 / n$ and $\beta_{n}=\epsilon_{n}=1 / n^{2}$. Then $\left\{\alpha_{n}\right\},\left\{\beta_{n}\right\},\left\{\epsilon_{n}\right\}$, and $\left\{\sigma_{n}\right\}$ satisfy conditions (i) and (ii) of Theorem 14. But we note that $\alpha_{n} / \beta_{n}=n \rightarrow \infty$.

Remark 16. In the iterative scheme of Theorem 14, the first iterative step $y_{n}=\alpha_{n} f\left(y_{n}\right)+\beta_{n} x_{n}+\left(\left(1-\beta_{n}\right) I-\alpha_{n} A\right)(I-$ $\left.\epsilon_{n} F\right) T_{n} y_{n}$ is a predictor step and the second iterative step $x_{n+1}=\sigma_{n} f\left(y_{n}\right)+\left(I-\sigma_{n} A\right) T_{n} y_{n}$ is a corrector step. Hence our iteration process is the predictor-corrector method.

Remark 17. Theorem 14 extends and improves Theorem 3.1 of [10] to a great extent in the following aspects:

(i) $u$ is replaced by a fixed contractive mapping;

(ii) one continuous pseudocontractive mapping (including nonexpansive mapping) is replaced by a countable family of nonexpansive mappings;

(iii) condition $\alpha_{n} / \beta_{n} \rightarrow 0$ is weakened to the one $\alpha_{n} \rightarrow 0$ and $\beta_{n} \rightarrow 0$ as $n \rightarrow \infty$;

(iv) we add a strongly positive linear bounded operator $A$ and a strongly accretive and strictly pseudocontractive mapping $F$ in our iterative algorithm.

Theorem 18. Let $C$ be a nonempty closed convex subset of a uniformly smooth Banach space $X$ which has the weakly sequentially continuous duality mapping $J$. Assume that $C \pm$ $C \subset C$. Let $\left\{T_{i}\right\}_{i=0}^{\infty}$ be a countable family of nonexpansive mappings from $C$ to itself such that $\Omega=\bigcap_{i=0}^{\infty} \operatorname{Fix}\left(T_{i}\right) \neq \emptyset$. Let $F: C \rightarrow C$ be $\alpha$-strongly accretive and $\lambda$-strictly pseudocontractive with $\alpha+\lambda>1$, and let $f: C \rightarrow C$ be a fixed contractive mapping with contractive coefficient $\beta \in$ $\left(0, \gamma_{0}\right), \gamma_{0}=1-\sqrt{(1-\alpha) / \lambda}$. Let $A: C \rightarrow C$ be a $\bar{\gamma}$-strongly positive linear bounded operator with $\bar{\gamma} \beta<1$. For arbitrarily given $x_{0} \in C$, let the sequence $\left\{x_{n}\right\}$ be generated iteratively by

$$
\begin{aligned}
& x_{n+1} \\
& =\left(I-\beta_{n} F\right) T_{n} x_{n}+\beta_{n}\left[f\left(x_{n}\right)-\alpha_{n}\left(A f\left(x_{n}\right)-T_{n} x_{n}\right)\right],
\end{aligned}
$$$$
\forall n \geq 0,
$$

where $\left\{\alpha_{n}\right\}$ and $\left\{\beta_{n}\right\}$ are two sequences in $(0,1)$ satisfying the following conditions:

(i) $\lim _{n \rightarrow \infty} \alpha_{n}=\lim _{n \rightarrow \infty} \beta_{n}=0$ and $\sum_{n=0}^{\infty} \beta_{n}=\infty$;

(ii) $\sum_{n=1}^{\infty}\left|\beta_{n}-\beta_{n-1}\right|<\infty$ or $\lim _{n \rightarrow \infty} \beta_{n-1} / \beta_{n}=1$.

Assume that $\sum_{n=0}^{\infty} \sup _{x \in D}\left\|T_{n+1} x-T_{n} x\right\|<\infty$ for any bounded subset $D$ of $C$, let $T$ be a mapping of $C$ into itself defined by $T x=\lim _{n \rightarrow \infty} T_{n} x$ for all $x \in C$, and suppose that $\operatorname{Fix}(T)=$ $\bigcap_{i=0}^{\infty} \operatorname{Fix}\left(T_{i}\right)$. Then, $\left\{x_{n}\right\}$ converges strongly to a point $z$ of $\Omega$ such that $z$ is a unique solution in $\Omega$ to the VIP:

$$
\langle(F-f) z, J(z-p)\rangle \leq 0, \quad \forall p \in \Omega .
$$

Proof. First, since $A$ is a $\bar{\gamma}$-strongly positive linear bounded operator on $C$, from (11) we have

$$
\|A\|=\sup \{|\langle A u, J(u)\rangle|: u \in C,\|u\|=1\} .
$$

Let us show that $\left\{x_{n}\right\}$ is bounded. Indeed, since $\lim _{n \rightarrow \infty} \alpha_{n}=0$, without loss of generality, we may assume that $0<\alpha_{n} \leq \min \left\{\left(\gamma_{0}-\beta\right) / 2(1-\bar{\gamma} \beta),\|A\|^{-1}\right\}, \forall n \geq 0$. Take $p \in \Omega$. Then it follows that $p=T_{n} p, \forall n \geq 0$, and

$$
\begin{aligned}
x_{n+1}-p= & \left(I-\beta_{n} F\right) T_{n} x_{n}-\left(I-\beta_{n} F\right) T_{n} p \\
+ & \beta_{n}\left[\left(I-\alpha_{n} A\right) f\left(x_{n}\right)-\left(I-\alpha_{n} A\right) f(p)\right. \\
& \left.+\alpha_{n}\left(T_{n} x_{n}-p\right)\right] \\
& +\beta_{n}(f-F) p+\beta_{n} \alpha_{n}(I-A f) p .
\end{aligned}
$$

Hence we deduce the following $0<\alpha_{n} \leq \min \left\{\left(\gamma_{0}-\beta\right) / 2(1-\right.$ $\bar{\gamma} \beta),\|A\|^{-1}$ \} that

$$
\begin{aligned}
& \left\|x_{n+1}-p\right\| \\
& =\|\left(I-\beta_{n} F\right) T_{n} x_{n}-\left(I-\beta_{n} F\right) T_{n} p \\
& +\beta_{n}\left[\left(I-\alpha_{n} A\right) f\left(x_{n}\right)-\left(I-\alpha_{n} A\right) f(p)\right. \\
& \left.+\alpha_{n}\left(T_{n} x_{n}-p\right)\right] \\
& +\beta_{n}(f-F) p+\beta_{n} \alpha_{n}(I-A f) p \| \\
& \leq\left\|\left(I-\beta_{n} F\right) T_{n} x_{n}-\left(I-\beta_{n} F\right) T_{n} p\right\| \\
& +\beta_{n}\left[\left\|I-\alpha_{n} A\right\|\left\|f\left(x_{n}\right)-f(p)\right\|+\alpha_{n}\left\|T_{n} x_{n}-p\right\|\right] \\
& +\beta_{n}\|(f-F) p\|+\beta_{n} \alpha_{n}\|(I-A f) p\| \\
& \leq\left(1-\beta_{n} \gamma_{0}\right)\left\|x_{n}-p\right\| \\
& +\beta_{n}\left[\left(1-\alpha_{n} \bar{\gamma}\right) \beta\left\|x_{n}-p\right\|+\alpha_{n}\left\|x_{n}-p\right\|\right] \\
& +\beta_{n}\|(f-F) p\|+\beta_{n} \alpha_{n}\|(I-A f) p\| \\
& =\left[1-\beta_{n}\left(\gamma_{0}-\beta-\alpha_{n}(1-\bar{\gamma} \beta)\right)\right]\left\|x_{n}-p\right\| \\
& +\beta_{n}\|(f-F) p\|+\beta_{n} \alpha_{n}\|(I-A f) p\| \\
& \leq\left[1-\beta_{n}\left(\gamma_{0}-\beta-\frac{\gamma_{0}-\beta}{2(1-\bar{\gamma} \beta)}(1-\bar{\gamma} \beta)\right)\right]\left\|x_{n}-p\right\| \\
& +\beta_{n}\|(f-F) p\|+\beta_{n} \alpha_{n}\|(I-A f) p\| \\
& \leq\left(1-\frac{1}{2} \beta_{n}\left(\gamma_{0}-\beta\right)\right)\left\|x_{n}-p\right\| \\
& +\beta_{n}(\|(f-F) p\|+\|(I-A f) p\|) \\
& =\left(1-\frac{1}{2} \beta_{n}\left(\gamma_{0}-\beta\right)\right)\left\|x_{n}-p\right\| \\
& +\frac{1}{2} \beta_{n}\left(\gamma_{0}-\beta\right) \frac{2(\|(f-F) p\|+\|(I-A f) p\|)}{\gamma_{0}-\beta} \\
& \leq \max \left\{\left\|x_{n}-p\right\|, \frac{2(\|(f-F) p\|+\|(I-A f) p\|)}{\gamma_{0}-\beta}\right\} \text {. }
\end{aligned}
$$


By induction

$$
\begin{aligned}
& \left\|x_{n}-p\right\| \\
& \leq \max \left\{\left\|x_{0}-p\right\|, \frac{2(\|(f-F) p\|+\|(I-A f) p\|)}{\gamma_{0}-\beta}\right\}, \\
& \forall n \geq 0 .
\end{aligned}
$$

This implies that $\left\{x_{n}\right\}$ is bounded and so are $\left\{T_{n} x_{n}\right\},\left\{f\left(x_{n}\right)\right\}$ and $\left\{F T_{n} x_{n}\right\}$.

Now we claim that

$$
\lim _{n \rightarrow \infty}\left\|x_{n+1}-x_{n}\right\|=0
$$

Indeed, first of all, (70) can be rewritten as follows:

$$
\begin{gathered}
y_{n}=\left(I-\alpha_{n} A\right) f\left(x_{n}\right)+\alpha_{n} T_{n} x_{n}, \\
x_{n+1}=\left(I-\beta_{n} F\right) T_{n} x_{n}+\beta_{n} y_{n}, \quad \forall n \geq 0 .
\end{gathered}
$$

Observe that

$$
\begin{aligned}
& \left\|y_{n}-y_{n-1}\right\| \\
& =\|\left(I-\alpha_{n} A\right) f\left(x_{n}\right)+\alpha_{n} T_{n} x_{n} \\
& -\left(I-\alpha_{n-1} A\right) f\left(x_{n-1}\right)-\alpha_{n-1} T_{n-1} x_{n-1} \| \\
& =\| \alpha_{n}\left(T_{n} x_{n}-T_{n-1} x_{n-1}\right) \\
& +\left(\alpha_{n}-\alpha_{n-1}\right)\left(T_{n-1} x_{n-1}-A f\left(x_{n-1}\right)\right) \\
& +\left(I-\alpha_{n} A\right) f\left(x_{n}\right)-\left(I-\alpha_{n} A\right) f\left(x_{n-1}\right) \| \\
& \leq \alpha_{n}\left\|T_{n} x_{n}-T_{n-1} x_{n-1}\right\| \\
& +\left|\alpha_{n}-\alpha_{n-1}\right|\left\|T_{n-1} x_{n-1}-A f\left(x_{n-1}\right)\right\| \\
& +\left\|I-\alpha_{n} A\right\|\left\|f\left(x_{n}\right)-f\left(x_{n-1}\right)\right\| \\
& \leq \alpha_{n}\left(\left\|T_{n} x_{n}-T_{n} x_{n-1}\right\|+\left\|T_{n} x_{n-1}-T_{n-1} x_{n-1}\right\|\right) \\
& +\left|\alpha_{n}-\alpha_{n-1}\right|\left\|T_{n-1} x_{n-1}-A f\left(x_{n-1}\right)\right\| \\
& +\left(1-\alpha_{n} \bar{\gamma}\right) \beta\left\|x_{n}-x_{n-1}\right\| \\
& \leq \alpha_{n}\left(\left\|x_{n}-x_{n-1}\right\|+\left\|T_{n} x_{n-1}-T_{n-1} x_{n-1}\right\|\right) \\
& +\left|\alpha_{n}-\alpha_{n-1}\right|\left\|T_{n-1} x_{n-1}-A f\left(x_{n-1}\right)\right\| \\
& +\left(1-\alpha_{n} \bar{\gamma}\right) \beta\left\|x_{n}-x_{n-1}\right\| \\
& =\left(\beta-\alpha_{n}(\bar{\gamma} \beta-1)\right)\left\|x_{n}-x_{n-1}\right\| \\
& +\left|\alpha_{n}-\alpha_{n-1}\right|\left\|T_{n-1} x_{n-1}-A f\left(x_{n-1}\right)\right\| \\
& +\alpha_{n}\left\|T_{n} x_{n-1}-T_{n-1} x_{n-1}\right\| \text {, }
\end{aligned}
$$

and hence

$$
\begin{aligned}
& \left\|x_{n+1}-x_{n}\right\| \\
& =\|\left(I-\beta_{n} F\right) T_{n} x_{n}+\beta_{n} y_{n} \\
& \quad-\left(I-\beta_{n-1} F\right) T_{n-1} x_{n-1}-\beta_{n-1} y_{n-1} \|
\end{aligned}
$$$$
\leq \| \beta_{n}\left(y_{n}-y_{n-1}\right)+\left(\beta_{n}-\beta_{n-1}\right)\left(y_{n-1}-F T_{n-1} x_{n-1}\right)
$$

$$
+\left(I-\beta_{n} F\right) T_{n} x_{n}-\left(I-\beta_{n} F\right) T_{n-1} x_{n-1} \|
$$

$$
\begin{aligned}
\leq & \beta_{n}\left\|y_{n}-y_{n-1}\right\|+\left|\beta_{n}-\beta_{n-1}\right|\left\|y_{n-1}-F T_{n-1} x_{n-1}\right\| \\
& +\left(1-\beta_{n} \gamma_{0}\right)\left\|T_{n} x_{n}-T_{n-1} x_{n-1}\right\| \\
\leq & \beta_{n}\left\|y_{n}-y_{n-1}\right\|+\left|\beta_{n}-\beta_{n-1}\right|\left\|y_{n-1}-F T_{n-1} x_{n-1}\right\| \\
& +\left(1-\beta_{n} \gamma_{0}\right)\left(\left\|T_{n} x_{n}-T_{n} x_{n-1}\right\|+\left\|T_{n} x_{n-1}-T_{n-1} x_{n-1}\right\|\right)
\end{aligned}
$$$$
\leq \beta_{n}\left\|y_{n}-y_{n-1}\right\|+\left|\beta_{n}-\beta_{n-1}\right|\left\|y_{n-1}-F T_{n-1} x_{n-1}\right\|
$$$$
+\left(1-\beta_{n} \gamma_{0}\right)\left(\left\|x_{n}-x_{n-1}\right\|+\left\|T_{n} x_{n-1}-T_{n-1} x_{n-1}\right\|\right)
$$$$
\leq \beta_{n}\left[\left(\beta-\alpha_{n}(\bar{\gamma} \beta-1)\right)\left\|x_{n}-x_{n-1}\right\|+\left|\alpha_{n}-\alpha_{n-1}\right|\right.
$$$$
\times\left\|T_{n-1} x_{n-1}-A f\left(x_{n-1}\right)\right\|
$$$$
\left.+\alpha_{n}\left\|T_{n} x_{n-1}-T_{n-1} x_{n-1}\right\|\right]
$$$$
+\left|\beta_{n}-\beta_{n-1}\right|\left\|y_{n-1}-F T_{n-1} x_{n-1}\right\|
$$$$
+\left(1-\beta_{n} \gamma_{0}\right)\left(\left\|x_{n}-x_{n-1}\right\|+\left\|T_{n} x_{n-1}-T_{n-1} x_{n-1}\right\|\right)
$$$$
\leq \beta_{n}\left[\left(\beta-\alpha_{n}(\bar{\gamma} \beta-1)\right)\left\|x_{n}-x_{n-1}\right\|+\left|\alpha_{n}-\alpha_{n-1}\right| M\right.
$$$$
\left.+\alpha_{n}\left\|T_{n} x_{n-1}-T_{n-1} x_{n-1}\right\|\right]+\left|\beta_{n}-\beta_{n-1}\right| M
$$$$
+\left(1-\beta_{n} \gamma_{0}\right)\left(\left\|x_{n}-x_{n-1}\right\|+\left\|T_{n} x_{n-1}-T_{n-1} x_{n-1}\right\|\right)
$$$$
=\left[1-\beta_{n}\left(\gamma_{0}-\beta-\alpha_{n}(1-\bar{\gamma} \beta)\right)\right]\left\|x_{n}-x_{n-1}\right\|
$$$$
+M\left(\beta_{n}\left|\alpha_{n}-\alpha_{n-1}\right|+\left|\beta_{n}-\beta_{n-1}\right|\right)
$$$$
+\left(\beta_{n} \alpha_{n}+\left(1-\beta_{n} \gamma_{0}\right)\right)\left\|T_{n} x_{n-1}-T_{n-1} x_{n-1}\right\|
$$$$
\leq\left[1-\beta_{n}\left(\gamma_{0}-\beta-\frac{\gamma_{0}-\beta}{2(1-\bar{\gamma} \beta)}(1-\bar{\gamma} \beta)\right)\right]
$$$$
\times\left\|x_{n}-x_{n-1}\right\|+M\left(\beta_{n}\left|\alpha_{n}-\alpha_{n-1}\right|+\left|\beta_{n}-\beta_{n-1}\right|\right)
$$$$
+2\left\|T_{n} x_{n-1}-T_{n-1} x_{n-1}\right\|
$$$$
=\left[1-\frac{1}{2} \beta_{n}\left(\gamma_{0}-\beta\right)\right]\left\|x_{n}-x_{n-1}\right\|
$$$$
+M\left(\beta_{n}\left|\alpha_{n}-\alpha_{n-1}\right|+\left|\beta_{n}-\beta_{n-1}\right|\right)
$$$$
+2\left\|T_{n} x_{n-1}-T_{n-1} x_{n-1}\right\| \text {, }
$$ 
where $\sup _{n \geq 0}\left\{\left\|T_{n} x_{n}-A f\left(x_{n}\right)\right\|+\left\|y_{n}-F T_{n} x_{n}\right\|\right\} \leq M$ for some $M>0$ (it is easy to see that $\left\{y_{n}\right\}$ is bounded due to the boundedness of $\left.\left\{x_{n}\right\}\right)$. Utilizing Lemma 2, we conclude that $\left\|x_{n+1}-x_{n}\right\| \rightarrow 0$ as $n \rightarrow \infty$ from conditions (i)-(ii) and the property imposed on $\left\{T_{n}\right\}$.

Next let us show that

$$
\lim _{n \rightarrow \infty}\left\|x_{n}-T x_{n}\right\|=0
$$

Indeed, from (76), (77), and $\beta_{n} \rightarrow 0$, it follows that

$$
\begin{aligned}
& \left\|x_{n}-T_{n} x_{n}\right\| \leq\left\|x_{n}-x_{n+1}\right\|+\left\|x_{n+1}-T_{n} x_{n}\right\| \\
& \quad=\left\|x_{n}-x_{n+1}\right\|+\beta_{n}\left\|y_{n}-F T_{n} x_{n}\right\| \longrightarrow 0 \quad \text { as } n \longrightarrow \infty .
\end{aligned}
$$

That is,

$$
\lim _{n \rightarrow \infty}\left\|x_{n}-T_{n} x_{n}\right\|=0
$$

Also, it is clear that

$$
\left\|x_{n}-T x_{n}\right\| \leq\left\|x_{n}-T_{n} x_{n}\right\|+\left\|T_{n} x_{n}-T x_{n}\right\| .
$$

By Lemma 12, we conclude from (82) and (83) that (80) holds. Let $x_{t}=\left(I-\theta_{t} F\right) T x_{t}+\theta_{t}\left[f\left(x_{t}\right)-t\left(A f\left(x_{t}\right)-T x_{t}\right)\right]$. According to Theorem 11, we know that $\left\{x_{t}\right\}$ converges strongly to $z \in$ $\operatorname{Fix}(T)=\bigcap_{i=0}^{\infty} \operatorname{Fix}\left(T_{i}\right)=\Omega$, which is the unique solution in $\Omega$ to the VIP:

$$
\langle(F-f) z, J(z-p)\rangle \leq 0, \quad \forall p \in \Omega .
$$

Further, let us show that

$$
\limsup _{n \rightarrow \infty}\left\langle(f-F) z, J\left(x_{n}-z\right)\right\rangle \leq 0 .
$$

Indeed, take a subsequence $\left\{x_{n_{i}}\right\}$ of $\left\{x_{n}\right\}$ such that

$$
\begin{aligned}
\limsup _{n \rightarrow \infty} & \left\langle(f-F) z, J\left(x_{n}-z\right)\right\rangle \\
& =\lim _{i \rightarrow \infty}\left\langle(f-F) z, J\left(x_{n_{i}}-z\right)\right\rangle .
\end{aligned}
$$

Without loss of generality, we may assume that $x_{n_{i}} \rightarrow \tilde{x}$. Utilizing Lemma 5 we obtain from (80) that $\tilde{x} \in \operatorname{Fix}(T)$. Hence from (84) and (86) we get

$$
\limsup _{n \rightarrow \infty}\left\langle(f-F) z, J\left(x_{n}-z\right)\right\rangle=\langle(f-F) z, J(\tilde{x}-z)\rangle \leq 0 .
$$

As required, let us show that $x_{n} \rightarrow z$ as $n \rightarrow \infty$.
As a matter of fact, we observe that

$$
\begin{aligned}
& \left\|x_{n+1}-z\right\|^{2} \\
& =\|\left(I-\beta_{n} F\right) T_{n} x_{n}-\left(I-\beta_{n} F\right) T_{n} z \\
& +\beta_{n}\left[\left(I-\alpha_{n} A\right) f\left(x_{n}\right)-\left(I-\alpha_{n} A\right) f(z)\right. \\
& \left.+\alpha_{n}\left(T_{n} x_{n}-z\right)\right]+\beta_{n}(f-F) z+\beta_{n} \alpha_{n}(I-A f) z \|^{2} \\
& \leq \|\left(I-\beta_{n} F\right) T_{n} x_{n}-\left(I-\beta_{n} F\right) T_{n} z \\
& +\beta_{n}\left[\left(I-\alpha_{n} A\right)\left(f\left(x_{n}\right)-f(z)\right)+\alpha_{n}\left(T_{n} x_{n}-z\right)\right] \|^{2} \\
& +2 \beta_{n}\left\langle(f-F) z, J\left(x_{n+1}-z\right)\right\rangle \\
& +2 \beta_{n} \alpha_{n}\left\langle(I-A f) z, J\left(x_{n+1}-z\right)\right\rangle \\
& \leq\left[\left\|\left(I-\beta_{n} F\right) T_{n} x_{n}-\left(I-\beta_{n} F\right) T_{n} z\right\|\right. \\
& \left.+\beta_{n}\left(\left\|I-\alpha_{n} A\right\|\left\|f\left(x_{n}\right)-f(z)\right\|+\alpha_{n}\left\|T_{n} x_{n}-z\right\|\right)\right]^{2} \\
& +2 \beta_{n}\left\langle(f-F) z, J\left(x_{n+1}-z\right)\right\rangle \\
& +2 \beta_{n} \alpha_{n}\|(I-A f) z\|\left\|x_{n+1}-z\right\| \\
& \leq\left\{\left(1-\beta_{n} \gamma_{0}\right)\left\|T_{n} x_{n}-T_{n} z\right\|\right. \\
& \left.+\beta_{n}\left[\left(1-\alpha_{n} \bar{\gamma}\right) \beta\left\|x_{n}-z\right\|+\alpha_{n}\left\|T_{n} x_{n}-z\right\|\right]\right\}^{2} \\
& +2 \beta_{n}\left\langle(f-F) z, J\left(x_{n+1}-z\right)\right\rangle \\
& +2 \beta_{n} \alpha_{n}\|(I-A f) z\|\left\|x_{n+1}-z\right\| \\
& \leq\left\{\left(1-\beta_{n} \gamma_{0}\right)\left\|x_{n}-z\right\|\right. \\
& \left.+\beta_{n}\left[\left(1-\alpha_{n} \bar{\gamma}\right) \beta\left\|x_{n}-z\right\|+\alpha_{n}\left\|x_{n}-z\right\|\right]\right\}^{2} \\
& +2 \beta_{n}\left\langle(f-F) z, J\left(x_{n+1}-z\right)\right\rangle \\
& +2 \beta_{n} \alpha_{n}\|(I-A f) z\|\left\|x_{n+1}-z\right\| \\
& =\left[1-\beta_{n}\left(\gamma_{0}-\beta-\alpha_{n}(1-\bar{\gamma} \beta)\right)\right]^{2}\left\|x_{n}-z\right\|^{2} \\
& +2 \beta_{n}\left\langle(f-F) z, J\left(x_{n+1}-z\right)\right\rangle \\
& +2 \beta_{n} \alpha_{n}\|(I-A f) z\|\left\|x_{n+1}-z\right\| \\
& \leq\left[1-\beta_{n}\left(\gamma_{0}-\beta-\alpha_{n}(1-\bar{\gamma} \beta)\right)\right]\left\|x_{n}-z\right\|^{2} \\
& +2 \beta_{n}\left\langle(f-F) z, J\left(x_{n+1}-z\right)\right\rangle \\
& +2 \beta_{n} \alpha_{n}\|(I-A f) z\|\left\|x_{n+1}-z\right\| \\
& \leq\left[1-\beta_{n}\left(\gamma_{0}-\beta-\frac{\gamma_{0}-\beta}{2(1-\bar{\gamma} \beta)}(1-\bar{\gamma} \beta)\right)\right]\left\|x_{n}-z\right\|^{2} \\
& +2 \beta_{n}\left\langle(f-F) z, J\left(x_{n+1}-z\right)\right\rangle \\
& +2 \beta_{n} \alpha_{n}\|(I-A f) z\|\left\|x_{n+1}-z\right\|
\end{aligned}
$$




$$
\begin{aligned}
= & {\left[1-\frac{1}{2} \beta_{n}\left(\gamma_{0}-\beta\right)\right]\left\|x_{n}-z\right\|^{2} } \\
& +2 \beta_{n}\left\langle(f-F) z, J\left(x_{n+1}-z\right)\right\rangle \\
& +2 \beta_{n} \alpha_{n}\|(I-A f) z\|\left\|x_{n+1}-z\right\| \\
= & \left(1-\mu_{n}\right)\left\|x_{n}-z\right\|^{2}+\mu_{n} v_{n},
\end{aligned}
$$

where $\mu_{n}=(1 / 2) \beta_{n}\left(\gamma_{0}-\beta\right)$ and

$$
\begin{aligned}
& v_{n} \\
& =\frac{4\left(\left\langle(f-F) z, J\left(x_{n+1}-z\right)\right\rangle+\alpha_{n}\|(I-A f) z\|\left\|x_{n+1}-z\right\|\right)}{\gamma_{0}-\beta} .
\end{aligned}
$$

It can be easily seen from (85) and conditions (i) and (ii) that

$$
\sum_{n=0}^{\infty} \mu_{n}=\infty, \quad \limsup _{n \rightarrow \infty} v_{n} \leq 0
$$

In terms of Lemma 8 , we infer that $x_{n} \rightarrow z$ as $n \rightarrow \infty$.

Finally, we provide an example to illustrate Theorem 18.

Example 19. Let $X=\mathbf{R}^{2}$ with inner product $\langle\cdot, \cdot\rangle$ and norm $\|\cdot\|$ which are defined by

$$
\langle x, y\rangle=a c+b d, \quad\|x\|=\sqrt{a^{2}+b^{2}},
$$

for all $x, y \in \mathbf{R}^{2}$ with $x=(a, b)$ and $y=(c, d)$. Let $C=\{(a, a): a \in \mathbf{R}\}$. Clearly, $C$ is a nonempty closed convex subset of a uniformly smooth Banach space $X=\mathbf{R}^{2}$ such that $C \pm C \subset C$. Let $\left\{T_{n}\right\}_{n=0}^{\infty}$ be a countable family of nonexpansive mappings from $C$ to itself such that $\Omega=$ $\bigcap_{n=0}^{\infty} \operatorname{Fix}\left(T_{n}\right) \neq \emptyset$, for instance, putting $T_{n}=\left(1-1 / 2^{n+1}\right) T$ with $T=\left\{\begin{array}{ll}3 / 5 & 2 / 5 \\ 2 / 5 & 3 / 5\end{array}\right\}$. Then $\|T\|=1$ and $\left\|T_{n}\right\|=1-1 / 2^{n+1}, \forall n \geq 0$. It is clear that $T_{n}$ and $T$ are nonexpansive mappings with $\Omega=\bigcap_{n=0}^{\infty} \operatorname{Fix}\left(T_{n}\right)=\{0\} \neq \emptyset$, and $\left\{T_{n}\right\}$ satisfies the assumption in Theorem 18. Let $F: C \rightarrow C$ be $\alpha$-strongly accretive and $\lambda$-strictly pseudocontractive with $\alpha+\lambda>1$, and $f$ : $C \rightarrow C$ be a fixed contractive mapping with contractive coefficient $\beta \in\left(0, \gamma_{0}\right), \gamma_{0}=1-\sqrt{(1-\alpha) / \lambda}$, for instance, putting $S=\left\{\begin{array}{ll}2 / 3 & 1 / 3 \\ 1 / 3 & 2 / 3\end{array}\right\}, F=(1 / 2) S$, and $f=\left\{\begin{array}{ll}3 / 25 & 2 / 25 \\ 2 / 25 & 3 / 25\end{array}\right\}$, we know that $\|F\|=(1 / 2)\|S\|=1 / 2,\|f\|=1 / 5$ and that $F$ is a (1/2)-strongly accretive and (8/9)-strictly pseudocontractive mapping and $f$ is a $(1 / 5)$-contraction with $(1 / 5) \in\left(0, \gamma_{0}\right)$ and $\gamma_{0}=1 / 4$. Let $A: C \rightarrow C$ be a $\bar{\gamma}$-strongly positive linear bounded operator with $\bar{\gamma} \beta<1$; for instance, putting $A=(7 / 6) S$, we know that $A$ is a $(7 / 6)$-strongly positive linear bounded operator with $\bar{\gamma} \beta=(7 / 6) \times(1 / 5)<1$. In this case, from iterative scheme (70) in Theorem 18, we obtain that for any given $x_{0} \in C$,

$$
\begin{aligned}
x_{1}= & \left(I-\beta_{0} F\right) T_{0} x_{0}+\beta_{0}\left[f\left(x_{0}\right)-\alpha_{0}\left(A f\left(x_{0}\right)-T_{0} x_{0}\right)\right] \\
= & \left(1-\frac{1}{2} \beta_{0}\right)\left(1-\frac{1}{2^{0+1}}\right) x_{0} \\
& +\beta_{0}\left[\frac{1}{5} x_{0}-\alpha_{0}\left(\frac{7}{6} \cdot \frac{1}{5} x_{0}-\left(1-\frac{1}{2^{0+1}}\right) x_{0}\right)\right] \\
= & {\left[\left(1-\frac{1}{2} \beta_{0}\right)\left(1-\frac{1}{2^{0+1}}\right)+\frac{1}{5} \beta_{0}\right.} \\
& \left.-\alpha_{0} \beta_{0}\left(\frac{7}{30}-\left(1-\frac{1}{2^{0+1}}\right)\right)\right] \quad x_{0} \in C .
\end{aligned}
$$

It can be readily seen that

$$
\begin{aligned}
x_{n+1}=[ & \left(1-\frac{1}{2} \beta_{n}\right)\left(1-\frac{1}{2^{n+1}}\right)+\frac{1}{5} \beta_{n} \\
& \left.-\alpha_{n} \beta_{n}\left(\frac{7}{30}-\left(1-\frac{1}{2^{n+1}}\right)\right)\right] x_{n}, \quad \forall n \geq 0 .
\end{aligned}
$$

We claim that $x_{n}$ converges to the unique point 0 in $\Omega$ if $\alpha_{n}=$ $(6 / 23) \beta_{n}$ and $\sum_{n=0}^{\infty} \beta_{n}=\infty$. Indeed, observe that

$$
\begin{aligned}
\left\|x_{n+1}\right\|= & {\left[\left(1-\frac{1}{2} \beta_{n}\right)\left(1-\frac{1}{2^{n+1}}\right)\right.} \\
& \left.+\frac{1}{5} \beta_{n}-\alpha_{n} \beta_{n}\left(\frac{7}{30}-\left(1-\frac{1}{2^{n+1}}\right)\right)\right]\left\|x_{n}\right\| \\
\leq & {\left[\left(1-\frac{1}{2} \beta_{n}\right)+\frac{1}{5} \beta_{n}-\alpha_{n} \beta_{n}\left(\frac{7}{30}-1\right)\right]\left\|x_{n}\right\| } \\
= & \left(1-\frac{3}{10} \beta_{n}+\frac{23}{30} \alpha_{n} \beta_{n}\right)\left\|x_{n}\right\| \\
= & \left(1-\frac{3}{10} \beta_{n}+\frac{23}{30} \cdot \frac{6}{23} \beta_{n} \beta_{n}\right)\left\|x_{n}\right\| \\
\leq & \left(1-\frac{3}{10} \beta_{n}+\frac{1}{5} \beta_{n}\right)\left\|x_{n}\right\| \\
= & \left(1-\frac{1}{10} \beta_{n}\right)\left\|x_{n}\right\| \leq \prod_{i=0}^{n}\left(1-\frac{1}{10} \beta_{i}\right)\left\|x_{0}\right\| .
\end{aligned}
$$

Thus, we conclude from $\sum_{n=0}^{\infty} \beta_{n}=\infty$ that $x_{n}$ converges to the unique point 0 in $\Omega$. It is clear that $z=0$ is a unique solution in $\Omega$ for the following variational inequality problem (VIP):

$$
\langle(f-F) z, J(p-z)\rangle \leq 0, \quad \forall p \in \Omega .
$$




\section{Conflict of Interests}

The authors declare that there is no conflict of interests regarding the publication of this paper.

\section{Acknowledgments}

This research was partially supported by the National Science Foundation of China (11071169), Innovation Program of Shanghai Municipal Education Commission (09ZZ133), and Ph.D. Program Foundation of Ministry of Education of China (20123127110002) for Lu-Chuan Ceng. This research was partially supported by a Grant from NSC for Chin-Feng Wen. This work was supported partly by the National Science Council of the Republic of China for Chin-Tzong Pang.

\section{References}

[1] G. Cai and S. Bu, "Approximation of common fixed points of a countable family of continuous pseudocontractions in a uniformly smooth Banach space," Applied Mathematics Letters, vol. 24, no. 12, pp. 1998-2004, 2011.

[2] L. C. Ceng, S. Schaible, and J. C. Yao, "Implicit iteration scheme with perturbed mapping for equilibrium problems and fixed point problems of finitely many nonexpansive mappings," Journal of Optimization Theory and Applications, vol. 139, no. 2, pp. 403-418, 2008.

[3] L. C. Ceng, P. Cubiotti, and J. C. Yao, "Strong convergence theorems for finitely many nonexpansive mappings and applications," Nonlinear Analysis: Theory, Methods and Applications, vol. 67, no. 5, pp. 1464-1473, 2007.

[4] L. C. Ceng and J. C. Yao, "Relaxed viscosity approximation methods for fixed point problems and variational inequality problems," Nonlinear Analysis: Theory, Methods and Applications, vol. 69, no. 10, pp. 3299-3309, 2008.

[5] L. C. Ceng and J. C. Yao, "Hybrid viscosity approximation schemes for equilibrium problems and fixed point problems of infinitely many nonexpansive mappings," Applied Mathematics and Computation, vol. 198, no. 2, pp. 729-741, 2008.

[6] H. Zegeye, E. U. Ofoedu, and N. Shahzad, "Convergence theorems for equilibrium problem, variational inequality problem and countably infinite relatively quasi-nonexpansive mappings," Applied Mathematics and Computation, vol. 216, no. 12, pp. 3439-3449, 2010.

[7] T. Suzuki, "Moudafi's viscosity approximations with MeirKeeler contractions," Journal of Mathematical Analysis and Applications, vol. 325, no. 1, pp. 342-352, 2007.

[8] Y. H. Wang and W. Xu, "Strong convergence of a modified iterative algorithm for hierarchical fixed point problems and variational inequalities," Fixed Point Theory and Applications, vol. 2013, article 121, 9 pages, 2013.

[9] J. S. Jung, "Iterative approaches to common fixed points of nonexpansive mappings in Banach spaces," Journal of Mathematical Analysis and Applications, vol. 302, no. 2, pp. 509-520, 2005.

[10] Y. Yao, Y. C. Liou, and R. Chen, "Strong convergence of an iterative algorithm for pseudocontractive mapping in Banach spaces," Nonlinear Analysis: Theory, Methods and Applications, vol. 67, no. 12, pp. 3311-3317, 2007.

[11] A. Moudafi and P.-E. Maingé, “Towards viscosity approximations of hierarchical fixed-point problems," Fixed Point Theory and Applications, vol. 2006, Article ID 95453, 10 pages, 2006.
[12] A. Moudafi, "Viscosity approximation methods for fixed-points problems," Journal of Mathematical Analysis and Applications, vol. 241, no. 1, pp. 46-55, 2000.

[13] M. Tian, "A general iterative algorithm for nonexpansive mappings in Hilbert spaces," Nonlinear Analysis: Theory, Methods and Applications, vol. 73, no. 3, pp. 689-694, 2010.

[14] L. C. Ceng, S. M. Guu, and J. C. Yao, "A general composite iterative algorithm for nonexpansive mappings in Hilbert spaces," Computers and Mathematics with Applications, vol. 61, no. 9, pp. 2447-2455, 2011.

[15] L. C. Ceng, Q. H. Ansari, and J. C. Yao, "Mann-type steepestdescent and modified hybrid steepest-descent methods for variational inequalities in banach spaces," Numerical Functional Analysis and Optimization, vol. 29, no. 9-10, pp. 987-1033, 2008.

[16] I. Yamada, "The hybrid steepest-descent method for variational inequality problems over the intersection of the fixed point sets of nonexpansive mappings," in Inherently Parallel Algorithms in Feasibility and Optimization and Their Applications, D. Butnariu, Y. Censor, and S. Reich, Eds., pp. 473-504, NorthHolland, Amsterdam, The Netherlands, 2001.

[17] H. K. Xu, "An iterative approach to quadratic optimization," Journal of Optimization Theory and Applications, vol. 116, no. 3, pp. 659-678, 2003.

[18] H. K. Xu, "Iterative algorithms for nonlinear operators," Journal of the London Mathematical Society, vol. 66, no. 1, pp. 240-256, 2002.

[19] H. K. Xu, "Viscosity approximation methods for nonexpansive mappings," Journal of Mathematical Analysis and Applications, vol. 298, no. 1, pp. 279-291, 2004.

[20] K. Goebel and W. A. Kirk, "Topics in metric fixed point theory," in Cambridge Studies in Advanced Mathematics, vol. 28, Cambridge University Press, 1990.

[21] K. Deimling, "Zeros of accretive operators," Manuscripta Mathematica, vol. 13, no. 4, pp. 365-374, 1974.

[22] Y. Song and R. Chen, "Convergence theorems of iterative algorithms for continuous pseudocontractive mappings," Nonlinear Analysis: Theory, Methods and Applications, vol. 67, no. 2, pp. 486-497, 2007.

[23] G. Cai and C. S. Hu, "Strong convergence theorems of a general iterative process for a finite family of $\lambda_{i}$-strict pseudocontractions in $q$-uniformly smooth Banach spaces," Computers and Mathematics with Applications, vol. 59, no. 1, pp. 149-160, 2010.

[24] K. Aoyama, Y. Kimura, W. Takahashi, and M. Toyoda, "Approximation of common fixed points of a countable family of nonexpansive mappings in a Banach space," Nonlinear Analysis: Theory, Methods and Applications, vol. 67, no. 8, pp. 2350-2360, 2007. 


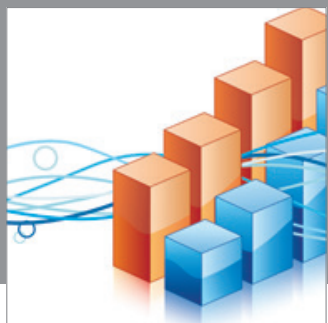

Advances in

Operations Research

mansans

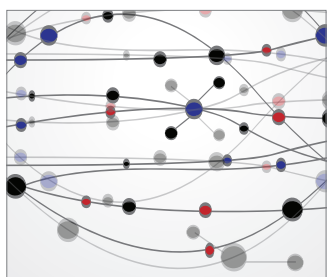

The Scientific World Journal
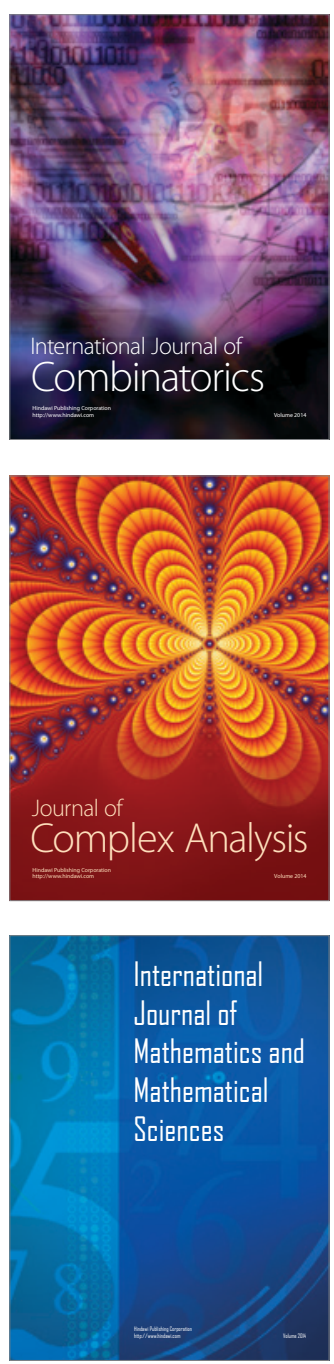
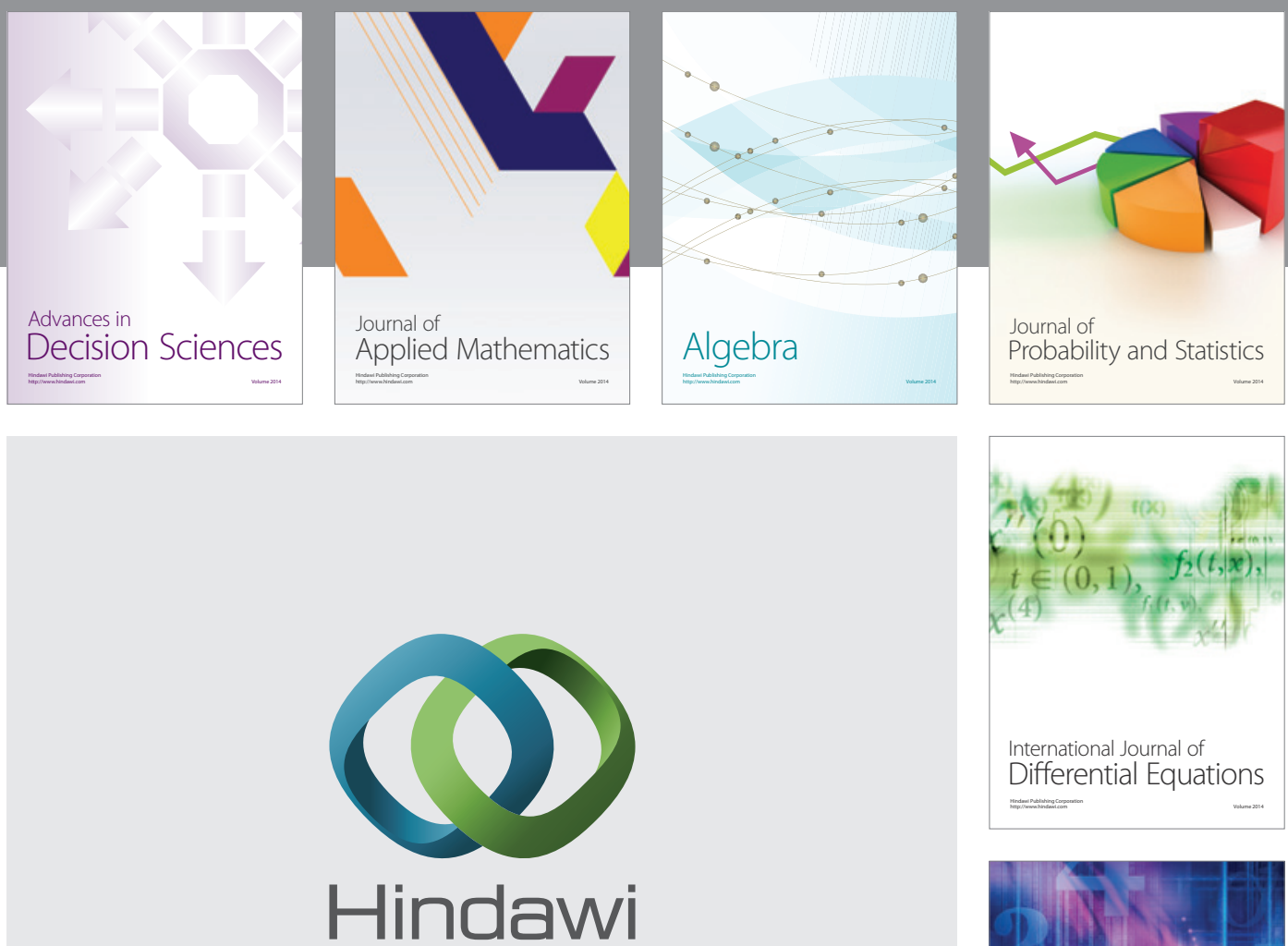

Submit your manuscripts at http://www.hindawi.com
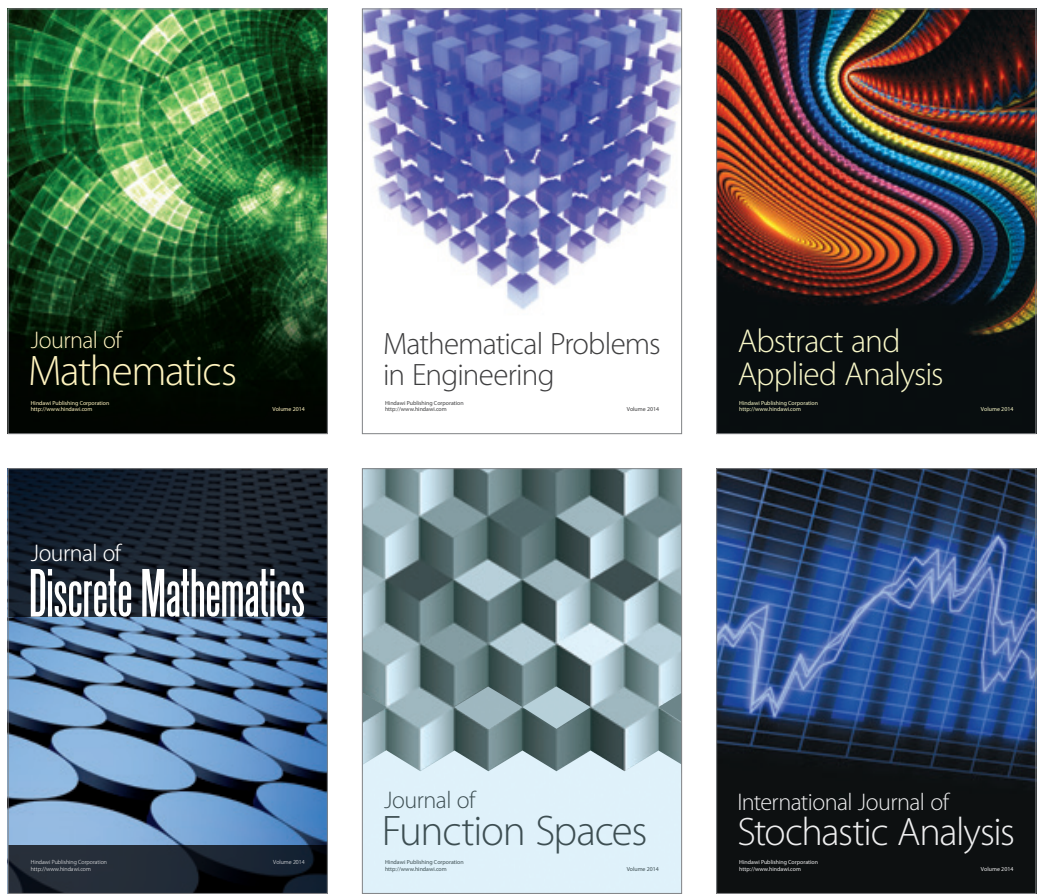

Journal of

Function Spaces

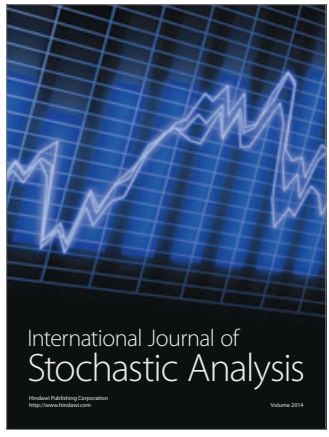

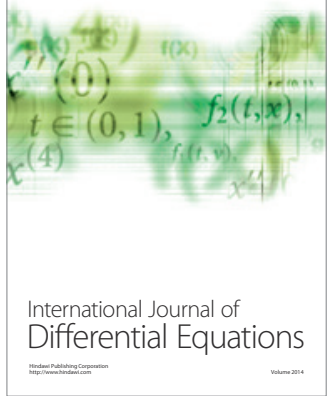
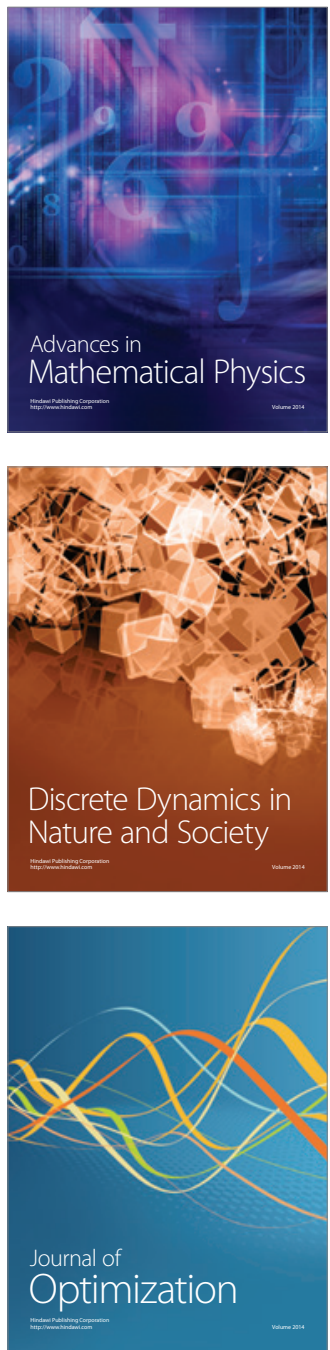